\title{
5. Aussprache und Schlussworte
}

\section{Primär- und Sekundärrechtsschutz im Öffentlichen Recht}

Vorsitzender (Bauer): Verehrte Kolleginnen und Kollegen, die vier Berichterstatter haben den zweiten Beratungsgegenstand unserer Tagung durch hervorragende Referate aufbereitet, fur die ich im Namen des Vorstands ganz herzlich danke. Für die Aussprache schlage ich vor, dass wir die Thematik im Schwerpunkt zunächst mehr aus der Sicht des deutschen Verwaltungsrechts erörtern und anschließend auf die mehr europarechtlichen und rechtsvergleichenden Aspekte übergehen. Ich hoffe auf ihr Einverständnis mit diesem Vorschlag und bitte Herrn Rupp um seinen Beitrag.

Rupp: Herr Vorsitzender, meine Kolleginnen und Kollegen, erlauben Sie mir drei kurze Anmerkungen. Zunächst: Der Unterschied zwischen der subjektrechtlichen Klagebefugnis und der Interessentenklage im verwaltungsgerichtlichen Anfechtungsprozess ist in der Praxis nicht so groß, wie man gelegentlich meinen könnte. Die Rechtsprechung des Bundesverwaltungsgerichts hat in jüngerer Zeit die Klagebefugnis des $\S 42 \mathrm{Abs} .2$ VwGO sehr weitherzig gehandhabt, so dass sie sich kaum mehr von der Klagebefugnis des Europarechts bzw. des französischen Rechts unterscheidet. Beide Positionen haben sich, wie ich meine, einander angenähert. In diesem Zusammenhang muss man auch sehen, dass zwischen dem Schutz subjektiver Rechte und der Validität der objektiven Rechtsordnung keine kontradiktorischen Gegensätze bestehen: Schon Rudolf von Jhering hat in seiner berühmten Schrift „Der Kampf ums Recht“" geradezu von einer moralischen Pflicht des Einzelnen gegenüber dem Gemeinwesen gesprochen, seine subjektiven Rechte durchzusetzen, um zur Realisierung des objektiven Rechts beizutragen. Zweite Bemerkung: Aus der Sicht der rechtswissenschaftlichen Systematik bestehen indes zwischen einer auf Durchsetzung subjektiver Rechte zielenden Klage und einer den Verstoß gegen die objektive Rechtsordnung rügenden Beanstandungsklage erhebliche Unterschiede. Die subjektivrechtliche Konzeption kommt meines Erachtens nicht so sehr in $\S 42 \mathrm{Abs} .2 \mathrm{VwGO}$, sondern in $\S 113$ Abs. 1 Satz 1 VwGO zum Ausdruck, wonach eine Anfechtungsklage dann begründet ist, wenn in eigenartiger Doppelbenennung der angefochtene Verwaltungsakt ,rechtswidrig und dadurch der Kläger in seinen Rechten verletzt ist". Eigenartig ist diese Doppelbenennung deshalb, weil die Verletzung jedes subjektiven Rechts als „rechtswidrig“ von der objektiven Rechtsordnung nicht mehr getragen wird. 
Jedenfalls stellt das derzeitige deutsche Verwaltungsrecht - wie der Zivilprozess - auf die Verfolgung subjektiver Rechte, genauer: materiellrechtlicher Reaktionsansprüche ab, was - wie die deutsche Verwaltungsprozessrechtslehre immer noch nicht erfasst hat - eine auf ebendiese subjektiven Rechte bezogene besondere Klagebefugnis als Prozessvoraussetzung sinnlos werden lässt. Jeder Zivilrichter würde nur verständnislos den Kopf schütteln, wenn von ihm etwa im Rahmen des § 823 I 2 BGB verlangt würde, das geltendgemachte subjektive Recht zweimal zu prüfen: Einmal summarisch als Prozessvoraussetzung und zum zweiten Mal gründlich und erschöpfend im Abschnitt über die Begründetheit der Klage. Auch der prozessökonomische Effekt solcher Unternehmungen ist - wie im einzelnen nachgewiesen - bestenfalls null. Dritte Bemerkung: Das Mitschleppen der besonderen Klagebefugnis des $\$ 42$ Abs. 2 VwGO ist ein Relikt vergangener Zeiten und lässt sich nur dadurch erklären, dass der deutsche Verwaltungsprozess bei seinem Beginn im 19. Jahrhundert eben nicht der Durchsetzung subjektiver Recht diente, sondern die objektive Rechtsordnung vor Verstößen zu bewahren suchte. Rudolf von Gneist, einer der wichtigsten Befürworter einer besonderen Verwaltungsgerichtsbarkeit in Deutschland, sah es geradezu als „civilistische petitio principii“" an, den Verwaltungsprozess in den Dienst subjektiver Rechte zu nehmen. Der Verwaltungsprozess habe ausschließlich die Bewahrung der verwaltungsrechtlichen Normenordnung zum Ziel; der Anfechtungskläger erhalte nur eine „formelle Parteirolle“, ohne dass ihm dadurch subjektive Rechte entstünden. Nur vor diesem Hintergrund war es jedenfalls überhaupt zu klären, dass vom Kläger eine gewisse Nähe, ein plausibles Interesse am Streitgegenstand, also eine prozessuale Klagebefugnis abverlangt wurde, um eine „Popularklage“ auszuschließen. Will man in Deutschland wieder zu diesem System des 19. Jahrhunderts zurückkehren, so müsste streng genommen als $\S 113$ Abs. 1 Satz 1 VwGO geändert, die Verletzung eines subjektiven Rechts gestrichen und die Klagebefugnis des $\S 42$ Abs. 2 VwGO auf verletzte subjektive Interessen bezogen werden. Wie sich dies allerdings mit den anderen - etwa auf Leistung oder Unterlassung gerichteten - verwaltungsgerichtlichen Klagen vertrüge, steht dahin. Vielen Dank.

Schoch: Herr Vorsitzender, meine sehr verehrten Kolleginnen und Kollegen. Ich möchte drei Bemerkungen machen: eine erste Bemerkung zum allgemeinen Zustand des Primärrechtsschutzes, eine zweite Bemerkung zur Europäisierung und den Vernetzungen zwischen Europarecht und nationalem Recht sowie eine dritte Bemerkung zur Durchsetzung des uns von Herrn Höfling präsentierten Innovationspotenzials im Sekundärrechtsschutz. 
Meine erste Bemerkung bezieht sich auf die Situation des primären Rechtsschutzes in Deutschland. Dazu muss man in aller Deutlichkeit sagen - und die Referate haben dies in begrüßenswerter Weise getan -, dass der Hauptsacherechtsschutz durch eine unglaubliche Atomisierung gekennzeichnet ist, dass das „Stammgesetz“, d.h. die VwGO, zunehmend mit Erfahrungen aus dem Sonderprozessrecht angereichert wird (bekanntestes Beispiel ist das Asylverfahrensrecht), dass wir daher Inkonsistenzen im Verwaltungsprozessrecht zu beobachten haben und dass weithin auch noch eine Insuffizienz des Rechtsschutzes herrscht. Das ist der beklagenswerte Zustand in weiten Teilen des Verwaltungsprozessrechts. Herrn Rupp würde ich - am Beispiel des zuletzt erwähnten Punktes leicht widersprechen wollen. Die Insuffizienz ist nicht nur eine Frucht gesetzgeberischer Aktivitäten, sondern auch der Rechtsprechung. Die Beispiele, die hier zum gerichtlichen Zugang, also zur Klagebefugnis, gebracht worden sind, waren vornehmlich dem Umwelt- und Planungsrecht entnommen. Schauen wir uns die privatisierten Bereiche an, sehen wir „Dreiecksverhältnisse“ im Wirtschaftsverwaltungsrecht. Das aktuell prominenteste Beispiel ist das kommunale Wirtschaftsrecht. Die Verwaltungsgerichte erkennen vielfach den einschlägigen Vorschriften nach wie vor keine Drittwirkung zu; es ist auch nicht so, dass die Grundrechte ergänzend herangezogen werden, so dass der verwaltungsgerichtliche Rechtsschutz im Ergebnis an dieser Stelle fast komplett ausfällt. In Rheinland-Pfalz hat der Verfassungsgerichtshof jetzt versucht, den Rechtsschutz etwas nach vorne zu bringen. Die Zivilgerichtsbarkeit ist bemüht, die bestehenden „Räume“ zu füllen (z.B. über § 1 UWG); allerdings kommen dann ganz andere Rationalitäten zum Tragen. In diesem hier nur exemplarisch herangezogenen Bereich sehe ich ein Versagen der Verwaltungsrechtsprechung; manifeste gesellschaftliche Konflikte werden nicht aufgegriffen und daher nicht im verwaltungsrechtlichen System verarbeitet. Meine Kritik richtet sich also an die Verwaltungsrechtsprechung.

Mein zweiter Punkt betrifft die Vernetzungen zwischen Europarecht und nationalem Recht. Insoweit ist meine These in Bezug auf den Sekundärrechtsschutz, dass die aus europarechtlicher Perspektive zu unserem Staatshaftungsrecht getroffenen Aussagen nur verdeutlichen, was wir über dessen beklagenswerten Zustand ohnehin bereits wissen und uns sowohl in der Analyse als auch in den Therapievorschlägen nur noch einmal unterstützen. Zum Primärrechtsschutz hat Herr Streinz auf den vorläufigen Rechtsschutz abgehoben. Dazu gibt es in der Tat reichhaltige Rechtsprechung des Europäischen Gerichtshofs, während zum Hauptsacherechtsschutz nur wenige Aussagen des Gerichtshofs z.B. zu Fristen, zur Kontrolldichte und mittelbar zur Klagebefugnis existieren. Ich bitte, 
den Blick einmal auf den Vernetzungsaspekt zu richten. Wir haben zum 01. 01. 2002 eine erneute Novellierung der VwGO zu erwarten. In dem vorliegenden Gesetzentwurf sehen die Pläne so aus, dass im Bereich des Hauptsacherechtsschutzes das Zulassungsverfahren für die Berufung bleibt; die zweite Instanz erreicht man - ohne Zulassung der Berufung durch das Verwaltungsgericht - nur über die Zulassungsbeschwerde. Im Bereich der Eilverfahren jedoch wird § 146 VwGO geändert; das Zulassungsverfahren fällt weg. Damit verfügen wir im Eilverfahren über zwei Instanzen. In der Praxis nun ist z.B. fast jeder zweite Fall, den ich am Verwaltungsgerichtshof Baden-Württemberg zu bearbeiten habe, schon jetzt im Bereich der Eilverfahren angesiedelt. In Zukunft werden viele Anwälte selbstverständlich die Chance zur Erreichung der zweiten Instanz suchen, dadurch noch stärker in den vorläufigen Rechtsschutz drängen und damit die Verfahren nach $\$ 80$ Abs. 5, 80a Abs. 3, 123 VwGO weiter aufwerten. Was den Instanzenzug betriff, haben wir also eine Asymmetrie zwischen Hauptsacheverfahren und Eilverfahren festzustellen.

Nun präsentiert uns Herr Streinz, dem ich insoweit in der Analyse zustimme, die Einwirkungen des Europarechts auf das deutsche Verwaltungsprozessrecht in einem nochmals aufgewerteten System des vorläufigen Rechtsschutzes. Sie haben, Herr Streinz, die Übertragung der Kriterien von Art. $242 \mathrm{EGV}$ auf $\S 80$ Abs. 5 VwGO erörtert; ergänzend müssten wir - Stichwort „Atlanta“-Entscheidung des Europäischen Gerichtshofs - die Übertragung der Maßstäbe von Art. 243 EGV auf $§ 123$ VwGO hinzufügen. In der Konsequenz dieser Einwirkungen des Europarechts haben wir bei Sachverhalten mit gemeinschaftsrechtlichem Bezug einen anderen Standard der Maßstäblichkeit als bei Sachverhalten mit rein innerstaatlichem Bezug. Frage: Kann das nationale Prozessrecht auf Dauer die beiden unterschiedlichen Standards durchhalten? Wir kennen die Problematik ja auch aus anderen Bereichen, z.B. $\$ 48 \mathrm{VwVfG}$. Oder ist es nicht so, dass wir einem fak tischen Europäisierungsdruck ausgesetzt sind, so dass wir diese Zweigleisigkeit bei der Maßstabsbildung auf Dauer nicht werden durchhalten können? Die Frage ist nun, Herr Streinz, auf die ich eine Antwort nicht kenne, woher nimmt der Europäische Gerichtshof sein - als rechtspolitisches Prinzip vielleicht anerkennenswertes - Kohärenzgebot, mit dem man das gesamte Verfahrensrecht im Grunde vereinheitlichen könnte, um Maßstäbe der eigenen Verfahrensordnung in das nationale Recht zu transponieren? Früher haben Diskriminierungsverbot und Effizienzgebot ausgereicht, um den Verwaltungsvollzug sicherzustellen. Was aber ist die rechtliche Grundlage, wenn wir diese faktische Europäisierung in den Blick nehmen?

Meine dritte Bemerkung, ganz knapp, zielt auf den Sekundärrechtsschutz. Ich stimme Ihnen, Herr Höfling, in der Analyse und weitgehend 
auch in der Therapie zu, habe aber nicht verstanden, warum Sie uns in These 16 ein Konzept anbieten, das auf Binnenpluralisierung setzt und nicht auf eine Konzentration des Rechtsschutzes. Meine Gegenthese ist: Nachdem sich der Bundesgerichtshof vielfach als "verfassungsresistent" gezeigt hat, erweist er sich in jüngster Zeit (und dies nicht nur in einem Fall) auch als "europarechtsresistent". Wenn Sie - die bestehenden Vorschläge haben Sie ja genannt - keine Rechtsschutzkonzentration vornehmen, garantiere ich Ihnen auf Grund von Erfahrungen aus anderen Bereichen, dass Sie Ihr Konzept nicht werden durchsetzen können. Welches sind die vorhandenen Möglichkeiten? Einmal gibt es den rechtspolitischen Vorschlag zur Streichung der Art. 19 Abs. 4 S. 2, 14 Abs. 3 S. 4, 34 S. 3 GG; Sie werden entgegnen, das sei politisch nicht durchsetzbar. Wenn wir aber nicht zu einer Rechtswegvereinheitlichung kommen, wird Ihr Konzept in der Praxis nicht durchsetzbar sein. Immerhin gibt es einen Gesetzentwurf Bayerns zur Änderung des $\S 40$ Abs. 2 VwGO, der in die richtige Richtung zielt. Die Alternative, die mir - wenn wir einmal die in Ihrem Referat erwähnte Kammerentscheidung vergessen - eingefallen ist, besteht in der Fortentwicklung der Rechtsprechung des Bundesverfassungsgerichts. Ist es denkbar, dass Ihr Konzept über den „Hebel“ des sekundären Grundrechtsschutzes, wie ich es einmal nennen möchte, durchgesetzt werden kann, was dazu führen könnte, dass z.B. auch Eingriffe in Art. 12 Abs. 1 GG auf dieser Ebene schutzbewehrt sind und im Schadensfall hinreichen bis zu Kompensationsansprüchen? Oder welchen Vorschlag können Sie machen, um Thr Konzept in der Praxis durchsetzen zu können? Vielen Dank.

Schuppert: Herr Vorsitzender, meine Damen und Herren, ich möchte versuchen, eine Brücke zu schlagen von der Thematik von heute zu der von gestern. Und von dem sehr schönen Referat von Herrn Höfling zu dem nicht minder schönen Referat von Herrn Alexy. Herr Alexy hat uns ja vorgetragen, und wer würde dem widersprechen wollen, dass wir eine Spielraumdogmatik brauchen. Eine solche Spielraumdogmatik aber muss - und jetzt bin ich bei Herrn Höfling - eingebettet sein in eine Kontrolldogmatik: denn es kann ja nicht nur darum gehen, Spielräume zu erforschen, so interessant sie auch sein mögen, sondern es geht ja auch darum, einen Blick darauf zu werfen, wie solche Spielräume ausgefültt werden und welche Fehler dabei passieren. Von der Spielraumdogmatik führt also ein gerader Weg zu einer Ausfüllungsdogmatik mitsamt einer spezifischen Fehlerquellenlehre - die Stichworte bei Herrn Alexy lauten: „Abwägungsfehler bei strukturellen Spielräumen“ und „Erkenntnisfehler bei Erkenntnisspielräumen“ - und damit letztlich zu den Vorschlägen von Herrn Höfling zur Entwicklung einer Kontrolldogmatik und der Sicher- 
stellung eines bestimmten Kontrollniveaus. Ich glaube, dass Herr Höfling vollkommen Recht hat, wenn er geltend macht, dass die Kontrolldogmatik auf Veränderungen in der Verwaltungswirklichkeit reagieren muss und insoweit unter Anpassungsdruck steht. Sie haben diese Verwaltungswirklichkeit zutreffend beschrieben und dabei auch auf so erfolgreiche Modebegriffe wie den der Verantwortungsteilung hingewiesen. Ich will jetzt aber nicht über Verantwortungsteilung reden, so schön ich diesen Begriff auch finde, auch nicht über Verantwortungsstufung oder Verantwortungsausdifferenzierung oder wie man sonst dies alles nennen könnte, sondern über den Tatbestand als solchen, der zu dieser Begriffsbildung animiert. Wir haben es bei der öffentlichen Aufgabenerfüllung offensichtlich mit einer Gemengelage von staatlichen, privaten und halbstaatlichen Akteuren zu tun - wie gerade auch Ihr schönes Beispiel des Transplantationsgesetzes mit einer Vielzahl von Akteuren belegt, die auf diesem Terrain mit verteilten Rollen agieren. Dies alles bedarf irgendwie der Kontrolle. Und ich teile auch Ihre Einschätzung, Herr Höfling, dass man innerhalb dieses - wie Herr Di Fabio es kritisch bezeichnet hat - Verantwortungsgebräus einer wie auch immer gearteten Verantwortungsidentifizierung bedarf. Aber wie kann man - das ist der Punkt, der mich interessiert - Verantwortung identifizieren, um sie sodann eindeutig zurechnen und kontrollieren zu können? Dazu möchte ich gerne einen Vorschlag machen: ein wichtiger Baustein für eine solche Verantwortungsidentifizierung könnte doch darin bestehen, dass man bereits bei der Strukturierung solcher Kooperationsbeziehungen rechtlichen Grund legt und mit Herrn Bauer die Entwicklung eines Verwaltungskooperationsrechts fordert. Ein solches Verwaltungskooperationsrecht mit dem Typ des Kooperationsvertrages als wichtigem Bestandteil könnte dabei helfen, die Rollen klarer zu machen, die die einzelnen Akteure wahrzunehmen haben. In einem solchen Verwaltungskooperationsrecht müsste festgehalten sein, welche Aufgaben der private Akteur erfüllt, welche Standards er dabei einzuhalten hat, wie die Qualitätssicherung der Aufgabenerfüllung gewährleistet wird, welche Kontrollrechte die Verwaltung hat und ob gegebenermaßen ein Rückholrecht besteht. Auf diese Weise würde bereits in einem sehr frühen Stadium eine deutliche Strukurierungsleistung der Rechtsordnung möglich und könnte so ein auskömmliches Kontrollniveau erreicht werden.

Breuer: Ich möchte zwei Punkte aufgreifen. Zunächst will ich einige grundsätzliche Bemerkungen zum System des Verwaltungsrechts und des Rechtsschutzes machen, zum zweiten einige Worte zum Staatshaftungsrecht sagen.

Zum ersten: Das Verwaltungsprozessrecht, also der Rechtsschutz, ist 
eigentlich nur die Speerspitze des Verwaltungsrechts. Die Unterscheidung zwischen den verschiedenen verwaltungsrechtlichen Prozesstypen bleibt meines Erachtens an der Oberfläche haften, wenn wir uns nicht darüber klar werden, dass das Verwaltungsrecht in Deutschland seit dem vorigen Jahrhundert eine besondere Richtung gewählt hat, und diese unterscheidet sich nun einmal vom französischen Verwaltungsrecht, vom britischen Verwaltungsrecht und heute auch vom europäischen Verwaltungsrecht, das weitgehend den Mustern des französischen und neuerdings auch des britischen Rechts folgt. Die Schlüsselbegriffe sind meines Erachtens das Verwaltungsrechtsverhältnis und das subjektive öffentliche Recht. Sie lassen sich an dem Gegensatz zwischen einer konditionalen oder finalen Struktur des Verwaltungsrechts festmachen. Das deutsche Verwaltungsrecht folgt dem Modell der konditionalen Rechtsordnung, und diese ist quasi zivilistisch ausgestaltet. Man denkt in den Kategorien der Rechtsansprüche und der Verpflichtungen. Man denkt des weiteren in den Kategorien rechtsbegrifflicher Tatbestände und der Justiziabilität, so dass ein Gericht darüber zu entscheiden hat, ob die Anspruchsvoraussetzungen namentlich zugunsten des Einzelnen erfuillt sind und entsprechend eine Verpflichtung des Staates eingreift. Diese quasi zivilistische Struktur hat tiefreichende historische Gründe. Herr Rupp hat von Gneist genannt, aber wenn man von Gneist nennt, muss man sofort darauf hinweisen, dass er im 19. Jahrhundert eine Ausnahmeposition eingenommen hat. Wenn man von Otto Bähr, Carl Friedrich von Gerber zu Otto Mayer kommt und dann zu Walter Jellinek, stellt man fest, dass im deutschen Verwaltungsrecht der Individualrechtsschutz an den Ansprüchen des Einzelnen festgemacht worden ist und dass die Hoffnung des Deutschen immer darauf gerichtet war, dass die Gerichte seinen Ansprüchen nachgehen. Ganz anders ist das final strukturierte Verwaltungsrecht in Frankreich und in Großbritannien aufgezäumt. Dort enthält nämlich das Gesetz Aufträge an die Exekutive, und damit sind auch die schon gestern und heute zitierten Spielräume der Exekutive angesprochen. Hier ist eher Misstrauen gegenüber der gerichtlichen Einmischung in die politischen Vorgänge der Gesetzgebung und der Gesetzesausführung durch die Exekutive festzustellen. Wir haben es also mit einem politischen Verwaltungsrecht zu tun, das durchaus ein rechtsstaatliches Verwaltungsrecht ist, aber die starke Stellung der Exekutive in der Gesetzesausführung ist prägend. Im britischen Verwaltungsrecht sind natürlich ganz andere Prämissen festzustellen, aber es ist doch so, dass auch hier die Exekutive mit einem besonderen Auftrag versehen ist. Die Gesetze bedienen sich oft einer so genannten subjective language, also einer subjektivierten Sprache, die ebenfalls darauf zielt, dass nach Einschätzung der Verwaltung die Gesetze zu vollziehen sind. Der Bürger hat hier also, wenn Sie so wollen, 
eine Hilssfunktion, ja er wird funktionalisiert, wie mehrfach gesagt worden ist. Dies ist aber keine Erfindung des Europarechts, sondern das ist eine ganz konsequente Entwicklung, die man im englischen, im britischen und nun auch im europäischen Verwaltungsrecht feststellt. Ich will die Gegensätze nicht überzeichnen, man muss zur Annäherung kommen, damit hat Frau Epiney sicherlich recht. Aber man muss eben doch darauf hinweisen, dass es nicht nur um einige prozessuale Unterschiede, z.B. der Klagebefugnis und sonstiger prozessualer Prämissen, geht, sondern dass das Prozessrecht hier das materielle Verwaltungsrecht widerspiegelt. Ich fürchte deshalb, dass wir nicht um bestimmte Strukturentscheidungen herumkommen. Wollen wir eigentlich ein quasi zivilistisches, streng juristisches Verwaltungsrecht, oder wollen wir ein finales und letztlich politisches Verwaltungsrecht? Diese Frage muss man nicht für alle Streitigkeiten ein für allemal apodiktisch entscheiden, aber es ist doch eine Strukturentscheidung, an der man nicht vorbeikommt. Damit hängt zusammen, ob wir eigentlich die verfahrensrechtlichen Positionen stärken wollen, was Herr Erbguth gefordert hat, oder ob man bis zu einem gewissen Grade an materiell-rechtlichen, gerichtlich durchsetzbaren Positionen des Bürgers festhalten will. Hier ist meines Erachtens ein Synkretismus, also eine beliebige Vermischung der Ansätze, nicht möglich, insofern muss man Farbe bekennen. Damit hängt wiederum die Frage der gerichtlichen Kontrolldichte zusammen. Die großen Probleme, die wir im deutschen Verwaltungsrecht in den letzten Jahren haben, und die vielen Kompromisse, die wir auch schließen, beruhen darauf, dass wir immer größere Schwierigkeiten haben, den quasi zivilistischen, streng juristischen Ansatz durchzuhalten. Wir nähern uns da schon ausländischen Rechtsordnungen an, die Nähe zur Schweiz stelle ich ohnehin erfreut fest, und auch andere Rechtsordnungen sind vielleicht auf dem Wege dazu, ein Stück weit doch materielle Positionen aufzunehmen. Aber wir sind weit entfernt von einer einheitlichen harmonischen Lösung. Und es geht letztlich um die Frage „quis iudicabit?", in unserem Rechtsstaat ist es im Zweifel die Verwaltung oder sind es im Zweifel die Gerichte. Erst wenn wir diese Fragen geklärt haben, kann man sich der Problematik stellen, wie die prozessuale Speerspitze geschliffen und aufgesetzt werden soll. An alle Referenten geht die Frage, ob ein solcher Strukturwandel des materiellen Verwaltungsrechts und des Prozessrechts ohne ein parlamentarisches Gesetz oder am parlamentarischen Gesetz vorbei erfolgen kann. Ich habe hier meine erheblichen Zweifel, der Vereinheitlichungsprozess ist meines Erachtens nicht ohne den parlamentarischen Gesetzgeber möglich.

Zum Staatshaftungsrecht nur einige Worte: Es ist von einem desolaten Zustand gesprochen worden, und jeder von uns könnte das, zwar nicht 
mit der gleichen Eloquenz wie Herr Höfling, aber doch mit ähnlichen Argumenten, belegen. Das Verblüffende ist nur, dass diejenigen, die im Schrifttum vom desolaten Zustand des Staatshaftungsrechts sprechen, zugleich die prägnantesten und brilliantesten Lehr- und Handbücher zu diesem Gebiet verfassen. So schlecht kann also das gewachsene Staatshaftungsrecht eben doch nicht sein. Etwas von einem gewachsenen System steckt offenbar darin. Die Frage, die ich daran anschließen möchte, ist die, ob es eigentlich heute nach wie vor möglich, sinnvoll oder sogar notwendig ist, ein Staatshaftungsgesetz in Deutschland zu konzipieren und $\mathrm{zu}$ erlassen, wie es 1981 ja schon einmal vom parlamentarischen $\mathrm{Ge}$ setzgeber verabschiedet worden ist, dann aber an Kompetenzgründen gescheitert ist. Wenn es einen desolaten Zustand gibt, dann beruht er ja darauf, dass die Rechtsgrundlagen weitestgehend dem Richterrecht entnommen werden. Man könnte hier durchaus auf die Idee kommen, es noch einmal zu versuchen, insbesondere angesichts der geänderten Kompetenzgrundlagen. Ich bin aber wiederum nicht ganz so sicher, ob man einfach abschreiben kann, was in dem seinerzeit verabschiedeten Staatshaftungsgesetz stand. Sind die Einflüsse des Europarechts und die notwendigen Annäherungsprozesse gegenüber den anderen Rechtsordnungen in Europa notwendiger Anlass, das Konzept noch einmal zu überdenken und vielleicht doch ein neues Gesetz zu konzipieren?

Klein: Ich kann partiell an den Beitrag von Herrn Breuer anknüpfen, der ja in einigen Wendungen von der Funktionalisierung oder auch der Instrumentalisierung des Einzelnen im Rechtsschutzsystem gesprochen hat, eine Begriffswendung die zumindest in den beiden letzten Referaten von Herrn Streinz und Frau Epiney aufgetaucht ist. Nun könnte man dieser Begriffsfindung natürlich etwas Negatives beilegen. Wer lässt sich schon gerne instrumentalisieren? Und erst recht dann hat das etwas Negatives, wenn man in einer Art Sanktionskategorie gepresst wird, wie das hier im Gemeinschaftsrecht der Fall ist. Aber man muss sich doch fragen, ob das so ungerechtfertigt ist, diese „Instrumentalisierung“ oder „Funktionalisierung “, weil ja immer jede Rechtsordnung darauf aus sein muss, dass Rechtsverletzungen rückgängig gemacht werden. Welche Mechanismen sie dabei anwendet, ist eigentlich zweitrangig, auch wenn das natürlich zu Folgen in der rechtlichen Gestaltung führen kann. Und gerade in einer Rechtsordnung, die in einem so prekären oder jedenfalls sehr lange doch sehr prekären Verhältnis zu den nationalen Rechtsordnungen stand, liegt es nahe, dass man sich bestimmter Mechanismen bedient, um Rechtsverletzungen besser beikommen zu können. Insofern glaube ich, ist zu diesem Zweck die Inanspruchnahme Einzelner absolut gerechtfertigt. Natürlich liegt insofern die unmittelbare Wirkung der Richtlinie auf 
derselben Ebene wie die Francovich-Entscheidung, wobei ich sogar im Hinblick auf letztere, das sei nur in Parenthese bemerkt, weniger Probleme habe, als mit der im Grunde contra legem angenommenen unmittelbaren Wirkung der Richtlinie. Wenn wir nun aber einen Blick ins nationale Recht werfen, dann stellen wir fest, dass diese Instrumentalisierung durchaus auch dem nationalen, also gerade auch dem deutschen Recht nicht fremd ist. Herr Rupp hat schon auf die historischen Wurzeln der Verwaltungsgerichtsbarkeit hingewiesen, und auch wenn sich das anders entwickelt hat, ist das ein interessanter Ausgangspunkt. Und wenn wir die Verfassungsbeschwerde nehmen, dann wird ja immer wieder auch die objektive Funktion der Verfassungsbeschwerde betont, also die Verfassungsbeschwerde als Instrument gesehen, das objektive Recht zu schützen. Natürlich geht dies durch einen Filter der Rechtsverletzung oder der behaupteten Rechtsverletzung. Aber dass dies eigentlich darüber hinausgeht, sieht man an der gestern freilich kritisierten Rechtsprechung des Bundesverfassungsgerichts, dass wenn eine Verfassungsbeschwerde einmal zulässig ist, das Bundesverfassungsgericht sich jedenfalls die Freiheit nimmt, auszugreifen und auch andere Normen für verfassungswidrig zu erklären, die nicht unmittelbar von dem tangierten Recht erfasst sind. Allerdings kann diese Instrumentalisierung nur, so verstehe ich das jedenfalls, auf den primären Rechtsschutz, wenn man in der herkömmlichen Terminologie bleibt, bezogen und angewendet werden, denn nur beim primären Rechtsschutz geht es gerade um die Beseitigung der Rechtsverletzung selbst. Und in allen Rechtsordnungen, vom nationalen Recht bis zum Völkerrecht, ist die primäre Rechtsfolge eines Normverstoßes, also einer Rechtsverletzung, zunächst einmal die Beendigung der Rechtsverletzung und die Rückkehr zum Recht. Genau das ist es, und das habe ich auch dem Referat von Herrn Höfling entnommen, was sich unmittelbar aus der Rechtsverletzung ergeben kann.

Eine letzte Bemerkung, und das ist eine ganz konkret an Herrn Streinz im Hinblick auf seine These 25 gerichtete Frage. Vor dem Hintergrund dessen, was ich eben ausgefuihrt habe, würde ich gerne wissen, wie es zu verstehen ist, wenn es im zweiten Teil dieser These heißt, dass das Gebot effektiven Rechtsschutzes nicht eindimensional verstanden werden darf, sondern auch im Sinne der Sicherung der Funktionsfähigkeit der Gesamtgemeinschaft verstanden werden muss. Bedeutet das eine Abschwächung des Rechtsschutzes im Hinblick auf die Funktionsfähigkeit der Gesamtgemeinschaft? Soll sich die Funktionsfähigkeit des Gemeinschaftsrechts als Beschränkung des Rechtsschutzes in ganz spezifischer Weise auswirken oder ist damit nur etwas gemeint, was freilich ganz generell gilt und daher auch den nationalen Rechtsschutz einbeziehen würde, dass nämlich ein Rechtsschutzmechanismus nie zur Aufhebung 
der Rechtsordnung und ihrer Funktionsfähigkeit führen darf. Das wäre ja gewiss nicht das, was das Recht und der von ihm gewährleistete Schutz sinnvollerweise zum Inhalt haben können.

Sachs: Ich bedanke mich bei allen vier Referenten, deren Referate mich sehr bereichert haben, möchte aber hier nur auf Herrn Höfling näher eingehen, weil ich mich bei ihm vielleicht nicht ganz so weit aufs Eis begeben muss, wie vielleicht beim Europarecht oder beim Planungsrecht. Ich stimme mit Herrn Höfling weitestgehend überein, möchte aber ein paar Punkte ansprechen, in denen ich unsicher bin. Einmal zu These $8 \mathrm{~b}$ und $\mathrm{c}$. Ich habe Zweifel, ob wir den grundrechtlichen Abwehrrechten unmittelbar mehr entnehmen können, als die in Punkt 8 a genannten negatorischen Hilfsansprüche. Bei Punkt $\mathrm{c}$ würde ich allerdings wegen der Grundrechtsmittelbarkeit zustimmen, wenn er sich damit auf einen Verfassungsauftrag an den Gesetzgeber bezieht, wenn also erst die gesetzliche Regelung notwendig wäre, um dann die grundrechtsmittelbare Berechtigung zu schaffen. Zu These 11 dann noch ein Punkt. Gegenüber der dort vorgeschlagenen Anknüpfung an die Eingriffsdogmatik scheint mir fraglich, ob wirklich einheitliche Zurechnungskriterien für Unterlassungs- und Ersatzansprüche gelten können. Ich fürchte, dass doch Einengungen im Bereich des Schadensersatzes notwendig wären, die dann auch den negatorischen Schutz, den wir ja weiterentwickelt haben bis in die Beeinträchtigungen mittelbarer und faktischer Art hinein, zurückdrängen müssten. Der Gesetzgeber muss beim Ersatzanspruch wohl doch Gestaltungsfreiheit haben, die ihm im übrigen ja fehlt. Ich sehe das Verhältnis von negatorischen und Ersatzansprüchen ähnlich wie bei den Eingriffen von Privatpersonen. Das sage ich auch mit Blick auf grundrechtliche Schutzpflichten, die ja möglicherweise auch für das Privatrecht parallele Konsequenzen nach sich ziehen müssten. Zuletzt zu These 14: Die Kompetenzeinweisung zugunsten der Judikative müsste ja wohl trotz Art. 74 Abs. $1 \mathrm{Nr} .25$ noch bundesstaatlich aufgeschlüsselt werden, also auf Bundes- und Landesgerichte verteilt werden, zumal es ja doch noch Länder gibt, deren Gesetze nicht ganz so unzureichend sind, wie das dem gemeindeutschen "Gewohnheitsunrecht" entspricht. Ich habe dies alles möglichst wörtlich abgelesen, um einmal zu testen, was dann bei der Niederschrift noch an Korrekturen notwendig sein wird.

Maurer: Herr Vorsitzender, meine Damen und Herren, ich möchte kurz auf vier Punkte eingehen, werde mich aber kurz fassen. Zunächst große Zustimmung zum Referat von Herrn Höfling, übrigens auch zu den anderen drei Referaten, aber ich möchte in erster Linie zu dem Referat von Herrn Höfling Stellung nehmen. Ich finde die Begründung des Ent- 
schädigungsanspruchs aus den Grundrechten überzeugend. Man könnte geradezu von einer Dreistufentheorie sprechen. Die Grundrechte sind primär Abwehrrechte. Greift der Abwehranspruch nicht oder nicht mehr, weil der Eingriff erfolgt ist, dann kommt gleichsam auf der zweiten Stufe der Beseitigungsanspruch oder Folgenbeseitigungsanspruch zum Zuge, der auf die Beseitigung der Folgen des Eingriffs und die Wiederherstellung des vorherigen Zustandes zielt. Der Folgenbeseitigungsanspruch ist bekanntlich nach 1945 von Herrn Bachof - ich glaube, er ist sogar hier im Saal - entwickelt worden. Er hat sich auch rasch durchgesetzt. Fraglich und strittig blieb allerdings lange die Begründung. Inzwischen wird ganz überwiegend die Auffassung vertreten, dass er sich aus den Grundrechten ergibt. Vom Folgenbeseitigungsanspruch oder Beseitigungsanspruch ist es nur noch ein Schritt zur nächsten Stufe, zum Entschädigungsanspruch. Wenn der Eingriff und seine Folgen nicht mehr korrigiert werden können, muss eben ein Ausgleich in Geld geleistet werden. Dieser Grundgedanke bestimmte übrigens auch die frühere Rechtsprechung des BGH zur Entschädigung wegen enteignungsgleichen Eingriffs. Das Gericht hatte zunächst an Art. 14 Abs. 3 GG angeknüpft, zog dann aber immer mehr Art. 14 GG insgesamt zur Begründung heran. Irritiert durch die Rechtsprechung des BVerfG leitet es nunmehr die Entschädigung wegen enteignungsgleichen Eingriffs aus dem allgemeinen Aufopferungsanspruch ab. Diese Wendung war nicht erforderlich und ist nicht überzeugend. Verfehlt ist auch die Beschränkung des BGH auf die Entschädigung wegen Eigentumsbeeinträchtigungen. Konsequenterweise müssten alle erfolgten und nicht mehr korrigierbaren Eingriffe in grundrechtliche Rechtspositionen im Rahmen des Möglichen entschädigt werden.

Noch eine kurze Bemerkung zu Art. 34 GG. Er wird relativ kleingeschrieben, was vielleicht daran liegt, dass er zwischen dem Staatsrecht und dem Verwaltungsrecht liegt. Nach der Rechtsprechung und der wohl herrschenden Lehre ist er lediglich eine Haftungsüberleitungsnorm, indem er den den Beamten treffenden Haftungsanspruch des $\$ 839$ BGB auf den Staat überleitet. Dass dies nicht oder nicht mehr stimmt, zeigt schon die Tatsache, dass sich beide Vorschriften nicht mehr voll entsprechen. Denn Art. 34 GG geht einerseits über § 839 BGB hinaus und bleibt andererseits hinter ihm zurück. Die Überleitungsthese, die in historischer Sicht richtig sein mag, wird der wirklichen Bedeutung des Art. 34 GG im verfassungsrechtlichen Kontext nicht mehr gerecht. Er stellt eine selbständige verfassungsrechtliche Haftungsnorm dar, die durch die Bezugnahme auf den traditionellen Amtshaftungsanspruch lediglich ergänzt und konkretisiert wird. Verfassungssystematisch stehen Art. 19 Abs. 4 GG und Art. 14 GG in engem Zusammenhang, da sie primären bzw. se- 
kundären Rechtsschutz gewährleisten und damit die in den Grundrechten verankerten subjektiven Rechte des Bürgers absichern. Sie bedürfen allerdings noch der gesetzlichen Ausgestaltung.

Dann noch ein dritter Gesichtspunkt: Es war viel von Primärrechtsschutz und Sekundärrechtsschutz die Rede, jedoch weniger vom Verhältnis beider, gleichsam vom Scharnier zwischen beiden. Warum trägt der Bürger durchweg die Anfechtungslast und das Anfechtungsrisiko, möglicherweise über mehrere Instanzen, wenn die Verwaltung den Fehler gemacht hat? Wo liegt die Zumutbarkeitsgrenze? Könnte man nicht in der Entschädigungsforderung zugleich den (eventuellen) Verzicht auf die betroffene Rechtsposition sehen? Im Enteignungsrecht hat ohnehin der freihändige Erwerb den Vorrang. Der Nassauskiesungsbeschluss des BVerfG, der sich fuir den strikten Vorrang des Primärrechtsschutzes aussprach, betraf einen relativ einfachen Fall. Fraglich bleibt die vom BVerfG intendierte und von der herrschenden Lehre ohne weiteres übernommene, ja sogar noch ausgedehnte Verallgemeinerung dieser Entscheidung.

Schließlich noch ein vierter und letzter Gesichtspunkt: Etwas knapp weggekommen ist die dogmatische Verankerung des gemeinschaftsrechtlichen Haftungsanspruches, der im Frankovich-Urteil des Europäischen Gerichtshofs erstmals präsentiert wurde. Die rechtsdogmatische Verankerung ist von erheblicher Bedeutung. Es gibt zwei Alternativen. Nach der ersten Alternative bildet der gemeinschaftsrechtliche Staatshaftungsanspruch einen selbständigen Anspruch, so etwa der Bundesgerichtshof. Neben den deutschen Haftungsansprüchen ist dann ggf. zusätzlich noch der gemeinschaftsrechtliche Haftungsanspruch zu prüfen. Nach der zweiten Alternative wird der gemeinschaftsrechtliche Haftungsanspruch in das deutsche Haftungssystem integriert. Einfacher mag die erste Alternative sein, besser ist die zweite Alternative. Sie hätte den Vorteil, dass es innerstaatlich bei einem einheitlichen, allerdings gemeinschaftsrechtlich überlagerten und modifizierten Haftungssystem bliebe. Zudem könnten durch die gemeinschaftsrechtlichen Impulse Defizite des deutschen Staatshaftungsrechts abgebaut und überwunden werden. Es sei nur auf das bereits erwähnte sog. legislative Unrecht oder auf das Subsidiaritätsprinzip im Amtshaftungsrecht verwiesen.

Schachtschneider: Schönen Dank, Herr Vorsitzender, liebe Kolleginnen und Kollegen! Die Freiheit wird durch allgemeine Gesetze verwirklicht. Also ist jede Gesetzesverletzung eine Verletzung der allgemeinen Freiheit, der Freiheit aller. Diese republikanische Logik müsste zur Popularklage führen. Das Unrecht in der Welt wird überall gespürt, hat Kant gesagt. René Marcic hat die Popularklage, welche von Juristen vehement 
abgelehnt zu werden pflegt, befürwortet. Die Referate gingen alle in die Richtung, das subjektive Recht mehr in die Nähe der Popularklage zu rücken, insbesondere die Hinweise von Frau Epiney auf die Praxis in Frankreich. Allemal kann man vertreten, dass eine Klagebefugnis habe, wer durch eine Rechtsverletzung betroffen ist. Normative Lehren vom subjektiven Recht im Sinne der Schutzzwecklehre scheinen mir fragwürdig zu sein. Für die Dogmatik vom subjektiven Recht sind die Anstöße der europäischen Rechtsentwicklung hilfreich. Mit Hilfe des Begriffs des subjektiven Rechts wird nach wie vor große Politik gemacht, vornehmlich im Bereich der europäischen Integration. Natürlich denke ich an den Euro-Beschluss. Mit keinem Wort etwa hat das Bundesverfassungsgericht den Schritt in die dritte Stufe der Währungsunion für rechtens erklärt. Es hat vielmehr gesagt, die Eigentumsgewährleistung, dort entgegen dem Wortlaut Eigentumsgarantie genannt, gehe nicht so weit, ein subjektives Recht darauf zu geben, den objektiv-rechtlichen Gehalt, nämlich das Stabilitätsprinzip, das auch in Art. 14 GG enthalten ist, mittels der Verfassungsbeschwerde geltend zu machen, um eine andere Stabilitätspolitik zu erwirken. Ich erinnere an unsere gestrige Erörterung. Das politische Ermessen oder eben der politische Spielraum soll so weit gehen, dass bei Entscheidungen, welche ökonomische Erkenntnisse zugrunde legen und die der politischen Gestaltung dienen, Rechtsschutz nicht beansprucht werden könne, d.h. dass ein subjektives Recht auf eine bestimmte Politik, eine Politik des Rechts nämlich, nicht bestehe, dass vielmehr diese Politik Verantwortung des Parlaments und der Regierung sei. Ich hoffe, dass ich diese für den Rechtsstaat verheerende Sentenz in etwa richtig wiedergegeben habe. Mit derartigen Sätzen zum subjektiven Recht kann man den Rechtsschutz in den großen politischen Fragen gänzlich beseite schieben. Betroffen sind wir alle. Jedenfalls durch die Währungsunion. Allemal gibt die allgemeine Handlungsfreiheit ein subjektives Recht auf vertragsgemäße, also rechtmäßige Politik, auch auf eine Integrationspolitik des Rechts. Allerdings hätte die Rechtlichkeit große politische Folgen gehabt. Jederzeit muss die Politik der Moral huldigen, lehrt Kant, und mit der Moral meint er das Recht. Die Richter müssen die Verantwortung auch für die Politik übernehmen, wenn das Gemeinwesen ein Rechtsstaat sein soll. Das Problem quält die Rechtswissenschaft nun schon seit der Praxis der römischen Prätoren. Die Richtung, die Sie, Frau Epiney, wenn auch nur mit Zurückhaltung, angedeutet haben, weist auf den richtigen Weg. Die anderen Referate haben sich ebenfalls von der engen Schutzzwecklehre abgewandt. Auch die Menschenrechtsbeschwerde wegen der Verletzung des Menschenrechts auf freie Meinungsbildung des Art. 10 EMRK vor dem Volksentscheid über den Beitritt Österreichs zur Europäischen Union 1994 ist mit dem Verdikt 
gegen die Popularklage vor der Kommission des Europäischen Gerichtshofs für Menschenrechte zurückgewiesen worden. Ich habe damals den Beitritt Österreichs für eine Wiener Gruppe, unter anderem den Künstler Hundertwasser, angegriffen, weil sich viele Österreicher durch die Propaganda für die Mitgliedschaft in der Europäischen Union, welche mitnichten der Wahrheit entsprach, verletzt fühlten. Diese Menschenrechtsbeschwerde, über die damals nicht öffentlich gesprochen werden durfte, wurde mit dem Argument der Popularklage abgelehnt, weil schließlich jeder Österreicher betroffen sei. Das ist untragbar. Jeder war betroffen, also hatte jeder ein subjektives Recht auf Recht. Ich plädiere für eine weite Zulassung von Klagen gegen das Unrecht. Das Unrecht muss wirksam bekämpft werden können, weil es alle zu spüren bekommen. Herr Streinz, vielen Dank für Ihre präzisen und hilfreichen Ausführungen zum europarechtlichen Fragenkreis des primären und sekundären Rechtsschutzes. Eine kleine Anregung: Es kann doch nicht richtig sein, dass wir nach Art. 100 GG das Normenverwerfungsmonopol für nachkonstitutionelle und formelle Gesetze haben, dass aber jeder Richter und sogar jeder Beamte ebenfalls nachkonstitutionelle formelle nationale Gesetze außer Anwendung lassen darf, wenn er der Meinung ist, dass sie dem Europarecht, sei es das Primärrecht, sei es eine Verordnung oder eine Richtlinie, widersprechen. Das wird aus der unmittelbaren Anwendbarkeit und dem Vorrang des Europarechts gefolgert. Der nationale Gesetzgeber, welcher immerhin durch Wahlen legitimiert ist, kann mehr Respekt erwarten. So stark ist das Gemeinschaftsrecht, welches man sogar der lex-posteriorRegel zu unterwerfen versucht hat, nicht. Ich empfehle die Analogie zu Art. $100 \mathrm{GG}$. Die Verfassungsgerichte hätten die unklaren Gemeinschaftsrechtsfragen dem Europäischen Gerichtshof vorzulegen. Die Vorlagepflicht besteht, aber nur, wenn das Gemeinschaftsrecht klärungsbedürftig ist. Die nationale Vorschrift steht zur Disposition jedes Gerichts und jedes Amtes. Herr Höfling, herzlichen Dank für Ihren unterhaltsamen Beitrag, bei dem Thema eine bewundernswerte Leistung. Erlauben Sie mir eine Bemerkung. Der Sekundärrechtsschutz steht nicht zur Wahl. Der Primärrechtsschutz geht vor. Jeder Bürger, gerade als Bürger der Republik, hat die Pflicht, für das Recht zu sorgen, also auch die Pflicht, die Gerichte für den Primärrechtsschutz zu bemühen. Sein Recht auf Recht folgt spezifisch aus dem Rechtsstaatsprinzip, welches freilich eine untrennbare Einheit mit dem Freiheitsprinzip und damit auch mit den materialen Grundrechten bildet. Ich bitte aber zu bedenken, dass, wenn wir mit dem vollen Beseitigungsanspruch oder einem rechtsstaatlichen Rechtsherstellungsanspruch das Verschuldensprinzip des $§ 839$ BGB aus dem Sekundärrechtsschutz gegenüber dem Staat herausdrängen, sollte der Gesetzgeber bemüht werden, um diese bedeutsame Rechtsentwick- 
lung demokratisch zu legitimieren. Ich befürworte eine solche Entwicklung. Herzlichen Dank.

Engel: Herr Höfling hat ein flammendes Plädoyer für die Befreiung des Rechts der öffentlichen Ersatzleistungen aus seinen zivilistischen Fesseln gehalten. Nicht, dass er Schützenhilfe nötig hätte. Aber ich will trotzdem versuchen, aus einer institutionenökonomischen Perspektive noch weitere Argumente hinzuzufuigen. Ich glaube, dass das deshalb reizvoll ist, weil das zivile Haftungsrecht der Ort war, an dem die ökonomische Analyse des Rechts überhaupt entwickelt worden ist. An drei der Grundkategorien will ich zeigen, wie anders sich die Fragen im Recht der öffentlichen Ersatzleistungen stellen. Auch aus diesem Grunde liegt es deshalb nahe, die Rechtsmassen auch genetisch voneinander abzukoppeln. Drei konzeptionelle Bausteine will ich mir näher ansehen: das Verhältnis von Kompensationsfunktion und Anreizfunktion; die Erkenntnis, dass Haftungsrecht implizit Verfügungsrechte zuordnet; schließlich das berühmte Coase-Theorem.

Zunächst also die erste Einsicht. Anders als wir es in unseren Zivilrechtsvorlesungen normalerweise noch beigebracht bekommen haben, erfüllt Haftungsrecht unvermeidlich zwei Funktionen zugleich. Einmal soll es natürlich geschehenes Unrecht wiedergutmachen. Das ist die Kompensationsfunktion. Aber gleichzeitig hat die Androhung von Kompensation auch Vorwirkungen, genauer Anreizwirkungen. In unserem Zusammenhang müsste das heißen: Vom Staatshaftungsrecht, also der Gefahr, den Bürger entschädigen zu müssen, geht eine Anreizwirkung auf Beamte und Parlament aus. Aus Furcht etwas bezahlen zu müssen, lassen sie vom Bruch des öffentlichen Rechts ab. Zunächst kann man sich schon fragen, ob das überhaupt eine realistische Vorstellung ist. Es würde voraussetzen, dass diejenigen, die solche Entscheidungen zu treffen haben, eine Beschränkung des öffentlichen Budgets tatsächlich fühlen werden. Man kann zunächst einmal einwenden, wenn das zum ernsthaften Problem wird, können sie ja die Steuern erhöhen. Das ist unter den gegenwärtigen Verhältnissen allerdings wahrscheinlich keine besonders realistische Option. Denn die Staatsquote ist ohnehin schon so hoch, dass sie nicht mehr sonderlich leicht zu steigern ist. Diejenigen, auf die man dann vor allem zugehen müsste, nämlich die Unternehmen, können in Zeiten des Systemwettbewerbs relativ glaubhaft mit Abwanderung drohen. Aus faktischer Sicht ist also schon ein bisschen was dran, aber eben auch nicht mehr als ein bisschen. Das, was politische Entscheidungen oder Verwaltungsentscheidungen prägt, ist letztlich doch nicht der Blick darauf, dass man finanziellen Handlungsspielraum verlieren könnte. Vor allem gibt es aber einen normativen Einwand. Im privatrechtlichen Zusammenhang 
mag man sagen, gut, wenn das diese Anreizwirkungen hat, ist das eine erfreuliche Nebenwirkung. In geeigneten Zusammenhängen kann man die Anreizwirkungen vielleicht sogar zur Hauptwirkung machen. Im öffentlich-rechtlichen Zusammenhang ist es dagegen schlechterdings unerträglich sich vorzustellen, dass wir nicht offen auf die Bindung des Staats an das Recht setzen, sondern auf die indirekten Wirkungen, vermittelt durch die Folgen für den staatlichen Geldsäckel.

Zweite Einsicht, die uns die ökonomische Analyse des Zivilrechts vermittelt: Im Haftungsrecht ist die Zuordnung von Verfügungsrechten versteckt. Beim § 823 Abs. 1 BGB sieht man das besonders schön bei den sog. absoluten Rechten. Wenn man sich dann ein bisschen näher ansieht, was die Zivilrechtsprechung aus dem Haftungsrecht gemacht hat, entdeckt man, dass die Verfuigungsrechte keineswegs stets beim Opfer liegen. So bewirkt die Ausgestaltung von Verkehrssicherungspflichten etwa gar nicht so selten, dass das Verfügungsrecht beim Schädiger liegt. Dann enthält das Haftungsrecht implizit die Befugnis, bestimmte Schädigungshandlungen sanktionslos vorzunehmen. Wir tun das, weil uns letztlich der soziale Nutzen bestimmter Übergriffe auf die Privatsphäre größer erscheint. Wir rechtfertigen es damit, dass wir eine staatliche Verteilungspolitik haben, die die Zufallsopfer schließlich entschädigt. Können wir diesen Gedanken aber auf das Recht der öffentlichen Ersatzleistungen übertragen? Die erste, impulsive Reaktion ist vermutlich vorschnell. Der Staat ist souverän. Er kann die Zuordnung der Verfügungsrechte deshalb ganz offen ändern. Aber im Verfassungsstaat sind Gesetzgeber und erst recht Verwaltung bei der Veränderung der Zuordnung von Verfügungsrechten nicht frei. Sie sind vielmehr an die Verfassung gebunden. Wir könnten das öffentliche Haftungsrecht deshalb scheinbar als einen zusätzlichen Schutzmechanismus für die verfassungsrechtlichen Regeln interpretieren, die etwas darüber aussagen, ob Verfügungsrechte dem Bürger weggenommen und dem Staat zugeordnet werden können. Wenn man näher hinsieht, stimmt das aber nicht. Denn die verfassungsrechtlichen Grenzen einer Neuzuordnung von Verfügungsrechten ergeben sich ja vor allem aus den Grundrechten. Wir müssen uns deshalb an das erinnern, was uns gestern Herr Alexy so eindrucksvoll vorgeführt hat: Die Grundrechte haben eben gerade keine konditionale, sondern eine finale Struktur. Sie enthalten nur eine Prinzipienordnung. Diese Prinzipienordnung wird erst im Einzelfall durch Abwägung konkretisiert.

Letzter Punkt: das berühmte Coase-Theorem. Ronald Coase hat es am Beispiel des Funkenflugs entwickelt, der von einer Eisenbahn ausgeht, die durch Kornfelder fährt. Er insistiert darauf, dass die Verfuigungsrechte in diesem Fall reziprok sind. Diese These richtet sich gegen den juristischen Impuls: „Aha, die Bahn ist schuld“, sie muss dem Bauern für die ver- 
brannte Ernte Ersatz leisten. Coase hält das aus einer Effizienzperspektive nicht für zwingend. Man kann genauso sagen: Der Bauer ist schuld. Er weiß doch, dass da eine Bahnlinie durchführt. Warum baut er seine Ähren gerade an dieser Stelle an? Die rechtspolitische Forderung ist dann, dass man sich im Einzelfall ansehen soll, welche Lösung die geringeren sozialen Kosten hat. Aus diesem Blickwinkel kann es dann wirklich besser sein, wenn in der Nähe von Eisenbahnlinien von vornherein keine Kornfelder angelegt werden. Frage: Lässt sich das auf unsere öffentlichrechtliche Situation übertragen? Es müsste dann einen rechtlich abgesicherten Anspruch des Staats darauf geben können, dass der Bürger einem vernünftigen Interventionsanspruch des Staats vorbeugend ausweicht. Das ist etwas, was im Rechtsstaat schlechterdings undenkbar ist. Auch ein Blick auf die entwickelte ökonomische Analyse des parallelen Instituts zeigt also, dass es an der Zeit ist, die historischen zivilistischen Wurzeln des Recht der öffentlichen Ersatzleistungen zu kappen.

Püttner: Herr Vorsitzender, ich möchte beginnen mit Herrn Schachtschneider und will ihm widersprechen, was ihn nicht wundern wird. Ich meine, die Popularklage hat in meinen Augen nicht dieses schöne Gesicht, was Sie ihr zugeschrieben haben, sondern Popularklage kann natürlich auch heißen, dass bestimmte Interessen, dass vielleicht auch sogar Querulanten die Dinge bestimmen. Also ich würde deshalb sagen, wenn schon Popularklage im Interesse der Überprüfung bestimmter staatlicher Akte, dann aber bitte schön eine limitierte Popularklage in dem Sinn, dass ein besonderes Annahmeverfahren vorzuschalten wäre. Ich könnte mir vorstellen, dass dies ginge. Aber wie gesagt, im übrigen würde ich Frau Epiney sagen: Auch bei uns gibt es schon gewisse Auflockerungen dieses strengen Prinzips rein subjektiven Rechtsschutzes, nämlich wie mir aufgefallen ist, bei der Allgemeinverfügung. Bei der Allgemeinverfügung (Beispiel etwa Schließung einer Schule) wird das Recht jedes Elternpaares anerkannt, sich gerichtlich gegen diese Schulschließung zu wehren und das liegt eigentlich schon ziemlich ähnlich den Fällen, die Sie aus der Schweiz berichtet haben. Also vielleicht kann man auf diesem Wege noch etwas weiterfahren und damit den Rechtsschutz auch in Deutschland, ohne dass man gleich den $\S 42$ Abs. 2 VwGO abschaffen muss, etwas erweitern. Und ich bin auch der Auffassung, dass bei den Schutznormen kritisch nachgeforscht werden könnte, inwieweit man nicht doch auch da per Auslegung oder per gesetzlicher Präzisierung mehr Schutz einbauen könnte, als er bisher anerkannt wird. Nun also zum Föderalismus. Da besteht folgender Zusammenhang: Das Scheitern eines vernünftigen Sekundärrechts gegenüber dem Chaos, Herr Höfling, wie Sie es geschildert haben, hängt ja auch damit zusammen, dass es unserem föderalistischen 
System bisher nicht gelungen ist, diese Materie in den Griff zu bekommen. Sie erinnern sich an $\S 839$ BGB, ein einziger Klimmzug, um an der mangelnden Kompetenz des Reiches vorbeizukommen. Dann der Versuch mit dem Staatshaftungsgesetz, das ja dann am Kompetenzmangel gescheitert ist. Heute haben wir nun eine Bundeskompetenz, aber ich habe den Eindruck, dass das Nichtaufgreifen dieser Kompetenz auch wieder mit den föderalistischen Gegebenheiten zusammenhängt, denn Bund und Länder verständigen sich offensichtlich nicht auf eine Lösung (und wenn sie teuer ist, das ist ja auch schwer). Aber ich meine, wenn der Bundesstaat bei uns seine Hausaufgaben nicht macht, dann gerät er in Gefahr, und wenn wir ins Auge fassen, was im Augenblick von Brüssel alles gegen unser Staatssystem ins Werk gesetzt wird, - das fängt bei der Gewährträgerhaftung für Landesbanken und Sparkassen an und hört anderswo auf - dann ist diese Bedrohung doch für uns eigentlich ein Anlass, wenigstens unsere Hausaufgaben im Bundesstaat zu erledigen und endlich ein vernünftiges Sekundärrecht zu schaffen, das dann auch europaweit Anerkennung finden kann. Natürlich spielt dabei auch der BGH eine gewisse Rolle. Herr Schoch hat mit Recht darauf hingewiesen, dass der BGH gerne aushilft, wenn andere Möglichkeiten verweigert werden, wenn die Verwaltungsgerichte nicht genügenden Rechtsschutz gewähren. Aber in den Fragen des Sekundärrechts habe ich den Eindruck, dass der Starrsinn des BGH doch eine größere Rolle spielt und dass auch deshalb der Gesetzgeber aufgerufen ist, in diesem Punkte endlich eine vernünftige Ordnung zu schaffen.

Spannowsky: Sehr geehrter Herr Vorsitzender, sehr geehrte Kolleginnen und Kollegen, mit meiner Stellungnahme beziehe ich mich auf 4 Problemfelder, die die Referenten teilweise zum Gegenstand ihrer Thesen gemacht haben. Herr Erbguth hat als These formuliert, dass der verwaltungsprozessuale Rechtsschutz, den er mit eingriffsbereinigender Kraft verbindet, auf die vorgelagerten Entscheidungsebenen zu erstrecken sei. Als Beispiele führte er die Linienbestimmung und die Raumordnungspläne an. $\mathrm{Ob}$ diese These verallgemeinerungsfähig ist, ist indessen zweifelhaft, zumal sich die der These zugrunde liegende, aus der PlanUVP-Richtlinie abgeleitete Logik, wonach die Standortalternativenprüfung auf die Ebene der Planung vorgezogen wird, weil sie bei der Vorhabenzulassungsentscheidung zu spät kommt, nicht auf sämtliche anderen Verwaltungsverfahren übertragen lässt. Zum einen besteht nicht immer eine Stufung wie im Planungsbereich, zum anderen besteht die Gefahr, dass den vorgelagerten Entscheidungen, deren verfahrensmäßige Rechtmäßigkeit durch das Gemeinschaftsrecht gewährleistet werden soll, durch den vorgezogenen Primärrechtsschutz eine materiell selbständige 
Bedeutung beigemessen wird. Eine weitere Gefahr liegt darin, dass die verfahrensmäßige Abschichtungsproblematik auf den Verwaltungsrechtsschutz, der nach dem Vorschlag Erbguths ebenfalls gestuft werden müsste, ausgedehnt wird.

Auch soweit Herr Höfling für eine stärkere Konzentration unrechtskompensatorischer Entscheidungskompetenzen bei den Verwaltungsgerichten plädiert, halte ich dies für fragwürdig, wenn dabei eine Verlagerung der Zuständigkeit für Amtshaftungs- und Entschädigungssachen intendiert wird. Denn man muss sich dabei bewusst sein, dass die Verlagerung auch mit der Folge verbunden wäre, dass die Verwaltungsgerichte sich insbesondere auch mit zivilprozessualen Fragen der Beweislast sowie materiellrechtlichen Fragen des Schadensumfangs befassen müssten. Eine Lösung der Rechtswegproblematik könnte demgegenüber darin liegen, dass der Dualismus der Rechtsordnung, der sich in der Praxis in den Richterpersonen widerspiegelt, partiell dadurch aufgelöst wird, dass für Spezialzuständigkeiten, wie etwa Amts- und Staatshaftungsangelegenheiten, eine gemischte Senatsbesetzung, bestehend aus Zivil- und Öffentlichrechtlern geschaffen wird. Beispiele dafür gibt es in der Praxis bereits.

Das dritte Problemfeld, das ich ansprechen möchte, bezieht sich auf das denkbare Nebeneinander von Amtshaftungsansprüchen und Ansprüchen wegen enteignungsgleichen Eingriffs. Durch dieses Nebeneinander wird der Sekundärrechtsschutz dadurch gegen den Primärrechtsschutz mobilisiert, dass der richterrechtlich ausgeformte Anspruch wegen enteignungsgleichen Eingriffs auch dann noch gegeben sein kann, wenn der Amtshaftungsanspruch wegen der gesetzgeberischen Beschränkungen gemäß $\S 839$ Abs. 1 S. 2 und Abs. 3 BGB ausgeschlossen ist. Während der BGH eine Korrektur über die Anwendung des § 254 BGB erwägt, sollte $\S 839$ Abs. 1 S. 2 und Abs. 3 BGB analog angewendet werden. Dies dürfte der Rechtsprechungslinie des BVerfG, dem Primärrechtsschutz den Vorrang einzuräumen, eher entsprechen.

Das vierte Problemfeld, das ich zum Erörterungsgegenstand machen möchte, bezieht sich auf die These von Herrn Streinz, wonach der Vorrang des Primärrechtsschutzes auch gemeinschaftsrechtlich geboten sei. Diese These möchte ich zunächst nachdrücklich unterstützen, wenngleich ich meine, dass in der bisherigen Rechtsprechung des EuGH immer noch eine Dominanz des Sekundärrechtsschutzes angelegt ist. Denn soweit nach der bisherigen Rechtsprechung des EuGH auch dann, wenn wegen nicht fristgerechter Umsetzung einer drittbegünstigenden Richtlinie ein Staatshaftungsanspruch gegeben ist, kumulativ ein Amtshaftungsanspruch in dem Fall eröffnet ist, dass ein Beamter eine direkt wirkende Richtlinie unangewendet gelassen und statt dessen zum Nachteil eines Dritten noch nicht angepasstes, schärferes Landesrecht angewendet hat, 
ist der Sekundärrechtsschutz, der zu Sanktionszwecken gegen die Mitgliedstaaten eingesetzt wird, unangemessen verschärft. Mit einer derartigen Problematik war der 6. Zivilsenat des OLG Zweibrücken, dem ich angehöre, in einem Fall befasst, in dem überhöhte Gebühren für die Durchfiihrung von Fleischhygienekontrollen erhoben worden sind. Die Klage war, nachdem die Gebührenbescheide aufgehoben waren, zur Geltendmachung des eingetretenen Zinsschadens auf einen Amtshaftungsanspruch wegen Nichtbeachtung des Anwendungsvorrangs des Gemeinschaftsrechts durch den Landesbeamten gestützt. Daneben wurde ein Staatshaftungsanspruch gegen das Land wegen nicht fristgerechter Richtlinienumsetzung geltend gemacht. Nachdem der EuGH inzwischen eine Staatshaftung wegen legislativen Unrechts infolge nicht fristgerechter Umsetzung von drittbegünstigenden EG-Richtlinien anerkannt hat, stellt sich die Frage, ob eine danebenstehende, zusätzliche Amtshaftung, noch sachgerecht erscheint. Dass dies problematisch ist, machte der fragliche Fall deutlich, wo die spätere Zurücknahme der Direktwirkung der Ausgangsrichtlinie durch eine Änderungsrichtlinie vom zuständigen Verwaltungsgericht nicht wahrgenommen worden war und dem Landesbeamten im anschließenden Amtshaftungsprozess wegen Zinsschäden zum Vorwurf gemacht wurde, dass er trotz der Direktwirkung der Richtlinie weiterhin entgegenstehendes Landesrecht angewendet hat.

Bei den geschilderten Problemfeldern handelt es sich um Einzelprobleme mit struktureller Bedeutung, die nur einen Ausschnitt der das Themenfeld im Verhältnis zwischen Primär- und Sekundärrechtsschutz prägenden komplexen Problematik abbilden, die aber - wie ich meine bedacht werden sollten. Ich bedanke mich für Ihre Aufmerksamkeit.

Meyer: Es ist nicht nur ein Problem der richtigen Bezeichnung, sondern auch ein Problem des richtigen Denkens, dass man nicht von Sekundärrechtsschutz spricht. Es handelt sich nicht um Rechtsschutz, sondern um Kompensationsrecht, Haftungsrecht oder wie immer man es bezeichnen will. Durch die Bezeichnung primär und sekundär suggeriert man die Vorstellung, es handle sich ungefähr um dasselbe, es werde nach demselben Muster abgehandelt, habe dieselbe Zielsetzung usw. Das ist aber ein großer Fehler, und daher sollte man die Terminologie aufgeben.

Beim nächsten Punkt will ich eine Innovation in die Verhandlung einbringen, nämlich einen teilweise mittelbaren Beitrag. Herr Pietzcker und Herr Wahl haben mich nämlich „beim Italiener“ ermächtigt zu sagen, dass sie in dem, was ich jetzt als Grundlage vortrage, mit mir völlig übereinstimmen. Herr Höfling, es wird Sie nicht wundern, dass es gegen ihr Referat gerichtet ist, weil es das innovationsfreundlichste war. Ich will auch die Verbindung zu gestern ziehen. Sie haben nichts weniger getan als 
der Maximierungstendenz der Grundrechte einen weiteren Schub zu verleihen. Jetzt sind sie nicht nur Bestandteil einer Wertordnung, die überall gilt und für unser gesamtes Handeln, sie sollen jetzt auch noch Anspruchsgrundlagen für Kompensationsansprüche werden, so habe ich Sie jedenfalls verstanden. Ich will Ihnen an dem Beispiel, das Sie selbst gebracht haben, zeigen, dass Sie ihre These nicht konsequent zu Ende gedacht haben und es auch gar nicht können. Das rechtswidrige Verbot einer Versammlung fuihrt nach Ihrer Meinung zu einem Kompensationsanspruch in Höhe der frustrierten Aufwendungen, also der unnützen Aufwendungen. Der Sinn des Versammlungsrechts, das verletzt worden ist, besteht aber nicht darin, keine unnützen Aufwendungen zu tätigen, sondern offensichtlich darin, einen demonstrativen Beitrag zur Meinungsbildung zu leisten. Wenn Sie wirklich aus dem Grundrecht eine Kompensation, die einigermaßen adäquat ist, verlangen würden, dann die, dass die verbietende Behörde den Slogan dieser Versammlung in den örtlichen Blättern veröffentlichen müsste. Und was machen Sie eigentlich, wenn das Briefgeheimnis verletzt worden ist. Nach Ihrer Theorie bestünde der Kompensationsanspruch bei einem Standardbrief bei DM 1,10, dem Preis für die Briefmarke. (Zwischenruf) Ja eben, er hat gesagt: die frustrierten Aufwendungen. Es gibt keine wirkliche Kompensation bei all den Grundrechten, deren Wert man nicht finanziell bemessen kann. Beim Eigentumsgrundrecht oder bei anderen Grundrechten mit berechenbaren Werten ist das kein Problem, bei den anderen aber wohl.

In diesem Zusammenhang möchte ich auf etwas anderes kommen. Niemand bedenkt, welche Konsequenz es hat, wenn wir für jedes rechtswidrige Handeln des Staates, das ja immer irgendwie auch grundrechtseingreifend ist, volle Kompensation vorschreiben. Ich kann Ihnen - und Herr Engel, das zeigt, wie dumm manchmal die Ökonomie des Rechts ist - ein Beispiel dafür geben, dass Beamte, die für ihre Fehler nicht bezahlen müssen, gleichwohl außerordentlich vorsichtig sind. Als § 48 VwVfG eingefuihrt wurde, bestimmte der Gesetzgeber, dass bei der Aufhebung von bestimmten Typen rechtswidriger Verwaltungsakte Entschädigung zu zahlen sei. Das hatte die Konsequenz, dass seitdem rechtswidrige Verwaltungsakte nicht mehr aufgehoben worden sind, wenn man nur befürchtete, dass Entschädigungsansprüche geltend gemacht werden würden. Das Verwaltungshandeln wird also von solchen Regelungen enorm beeinflusst. Wir tun im übrigen so, als sei es immer klar, was rechtmäßig oder rechtswidrig ist. In den meisten Fällen ist das unklar, und wir streiten uns darüber. Klarheit wird dann erst durch die richterliche Entscheidung herbeigeführt. Wenn Sie von Verfassungs wegen eine solche finanzielle Kompensation verlangen, wird die Konsequenz der Verwaltung sein, dass sie nur noch handelt, wenn sie ganz sicher ist, dass 
keiner von unseren Kollegen auf die Idee kommt zu sagen, das ist vielleicht doch rechtswidrig. Wollen wir das wirklich? Das wäre eine Einschränkung der politischen Möglichkeiten, vor der ich gestern schon gewarnt habe, sie wäre nur noch gravierender.

Rauschning: Es ist die Frage, ob wir in der Begrifflichkeit wirklich Herrn Meyer folgen sollten. Es ist natürlich die Frage, welche Begriffe wir wählen. Ich darf nur sagen, wenn Sie es alles ins Englische übertragen, dann merken Sie „remedies“ ist viel weiter, nicht wahr, natürlich wir übersetzen auch die Europäische Menschenrechtskonvention mit Rechtsbehelf statt mit Rechtsmitteln. Die Österreicher verwahren sich dagegen gegen unsere Begrifflichkeit und insoweit, wenn wir die etwas unschärferen Begriffe der anderen befreundeten Nationen sehen, dann ist halt die Frage, ob wir in der Begrifflichkeit wirklich unsere Schärfe durchhalten. Nein, ich wollte eigentlich ganz kurz das Wort nur nehmen, um Sie hinweisen zu können, auf eine reichhaltige Praxis in der Entschädigung für Menschenrechtsverletzungen, in der Human Rights Chamber of Bosnien Herzegovina. Wir haben wahrscheinlich deutlich über 100 Entscheidungen, in denen wir einschließlich Schmerzensgeld für Menschenrechtsverletzungen geben mussten infolge der europäischen Rechtsprechung, aber dort sicherlich etwas dezidierter als auch als Durchsetzungsmöglichkeit für die Bereinigung von Menschenrechtsverletzungen, wobei es sicherlich fraglich ist, ob man Verletzungen des Briefgeheimnisses entschädigen kann. Vieles andere kann man sicher nicht entschädigen. Und die Praxis ist ja dann auch so in Straßburg jedenfalls, dass man sagt, die Entscheidung, dass dieses rechtswidrig sei oder menschenrechtswidrig sei, ist eine hinreichende Wiedergutmachung. Auf der moralischen Seite ist das ja durchaus auch eine Möglichkeit der Wiedergutmachung. Ich wollte dies nur sagen, damit im gedruckten Teil ich die e-mail-Seite oder die Internet-Seite angeben kann, wo man dieses Material, das man sonst nicht findet, mit abdrucken kann. Ich danke Ihnen sehr.

Götz: Zuallererst möchte ich dem zustimmen, was Herr Meyer gesagt hat. Dann komme ich zum Europarecht. Hier habe ich den beiden europarechtlichen Referenten, Herrn Streinz und Frau Epiney, zu danken. Sie haben große europarechtliche Kompetenz bewiesen, und zwar nicht nur, was selbstverständlich wäre, durch die Zuverlässigkeit ihrer europarechtlichen Befunde, sondern auch durch das große Augenmaß, mit dem sie das nationale Recht, den nationalen Rechtsschutz, in den Blick genommen haben. Herr Streinz hat die Verfahrensautonomie der Mitgliedstaaten zutreffend hervorgehoben, und er hat am Schluss seines schönen 
Referates klargemacht, dass es um mehr geht als um einen kompetenzrechtlichen Grundsatz, nämlich insbesondere um die Bewahrung der Differenzierung, des Wertes und des Reichtums des nationalen Rechtsschutzes. Frau Epiney hat Rechtsvergleichung betrieben, die beste Methode, um sich im Europarecht vor Übertreibungen zu schützen. Die folgende Bemerkung betrifft die Klagebefugnis, über die Frau Epiney sehr eingehend berichtet hat. Die Referentin hat zutreffend aus den 1991 ergangenen Urteilen des EuGH zitiert, die die Umsetzung von Umweltschutzrichtlinien in Deutschland betrafen. Der EuGH verlangt die Klagbarkeit von Individualrechten, die sich aus der Verletzung der jeweiligen Umweltstandards ergeben und zwar auch, wenn es um Emissionswerte geht. Solche Rechte haben diejenigen Personen, die - so steht es dort - in ihrer Gesundheit beeinträchtigt sein könnten. Das kann dann, und darin folge ich Frau Epiney vollständig, die Zulässigkeit einer Interessentenklage zur Folge haben. Eine Interessentenklage, die man in Deutschland ohne weiteres an die Schutznormtheorie ,anlagern“ kann. Diese von Ihnen so bezeichnete normative Interessentenklage würde ich allerdings nicht in einem so strikten Gegensatz zu dem Erfordernis eines faktischen Betroffenseins des Klägers sehen. Ich würde von dem Erfordernis eines qualifizierten Betroffenseins sprechen. Geht es etwa um die Überschreitung der Emissionswerte, so kann, wie Sie auch ausgeführt haben, nicht etwa jeder klagen, sondern der Kläger muss ein faktisches Betroffensein darlegen oder, wie es vor Gericht heißt, „substanziieren“. Eine weitere und letzte Bemerkung, die ich machen möchte, betrifft die Kontrolldichte. Es stellt sich die Frage: Wird es unter dem Einfluss des Europarechtes zu einer Reduktion der Kontrolldichte kommen? Das kann man prophezeien, jedenfalls wenn es um die Anwendung von Normen des unmittelbar anwendbaren Europarechts einschließlich umgesetzter Richtlinien geht. Dazu ein Beispiel. Ein englisches Gericht hat kürzlich den EuGH in einem Vorabentscheidungsersuchen gefragt, welche Kontrolldichte es im Arzneimittelzulassungsrecht anzuwenden habe (Fall Upjohn, 1999). Ein Pharmaunternehmen klagte gegen den Widerruf der Arzneimittelzulassung. Die maßgebliche Norm war die umgesetzte Arzneimittelrichtlinie. Diese Norm stellte in einer Bestimmung, die man in Deutschland - aber kaum irgendwo sonst in Europa - als unbestimmten Rechtsbegriff beurteilen würde, auf den Maßstab der Unbedenklichkeit (Unschädlichkeit, Wirksamkeit) des Arzneimittels ab, und das englische Gericht fragte insbesondere, $o b$ es darüber selbst durch eigene Würdigung der herangezogenen wissenschaftlichen Beweise entscheiden müsse. Die Antwort des EuGH war sehr klar: Nein, das muss das Gericht nicht. Der EuGH führt aus, dass es ausreicht, wenn die nationalen Gerichte dieselben Kontrollstandards anwenden, die der EuGH selbst im Falle einer Nichtigkeits- 
klage gegen Entscheidungen der Kommission anwenden würde. Das bedeutet, etwas vereinfachend gesagt, dass alles, was die Anwendung jenes Begriffes betrifft, eine Ermessensfrage ist, und dass der Kontrollmaßstab, ich vereinfache etwas, nur die Frage nach offensichtlichen Ermessensfehlern ist. In der Entscheidung des EuGH ist offen geblieben, ob der nationale Richter etwa schärfere Kontrollmaßstäbe, eine höhere Kontrolldichte, anwenden könnte. Das kann er wohl, aber man muss nicht viel Phantasie haben, um zu folgern, dass dieses Urteil dennoch verstanden wird wie eine Aufforderung, es mit einer solchen Kontrolldichte, die nicht den deutschen Vorstellungen vom unbestimmten Rechtsbegriff entspricht, bewenden zu lassen.

Zuleeg: Herr Vorsitzender, ich habe vor, zu drei Referaten zu sprechen. Zunächst zu Ihnen, Herr Höfling. Ihr Referat schätze ich sehr. Ich bin auch grundsätzlich damit einverstanden, aber ich habe Schwierigkeiten, wenn Sie bei den Rechten nur oder vorwiegend die Grundrechte heranziehen. Ich meine, dass wir eine ganze Reihe von einfachen Rechten haben. Warum sollen sie nicht mit einbezogen werden? Es gibt die Leistungsrechte, die sozialen Rechte, es gibt politische Rechte. Der Europäische Gerichtshof hat in einem Gutachten, Gutachten EWR I, ausdrücklich gesagt, dass die Rechte Einzelner Bestandteil der Verfassung der Europäischen Gemeinschaft sind. Und ich meine, das sollte man auf Deutschland übertragen. Alle Rechte sind gleichmäßig zu schützen. Frau Epiney, ich habe mit Vergnügen Ihrem Referat gelauscht. Gegenwärtig haben wir allenfalls eine Zweiteilung bei der Klagebefugnis im deutschen Recht, nämlich auf der einen Seite die europäischen Rechte Einzelner und auf der anderen Seite die rein inländischen Positionen. Die erste Kategorie kann dadurch erfuillt werden, dass man $\S 42$ Abs. 2 Verwaltungsgerichtsordnung gemeinschaftskonform auslegt. Aber Sie wollen ja mehr, und dazu möchte ich Sie weiterhin aufrufen. Ich meine, dass der Rechtsstaat ausgebaut wird, wenn nach dem Vorbild des Gemeinschaftsrechts auch für die zweite Gruppe, für die inländischen Verfahren, das übernommen wird, was das Gemeinschaftsrecht vorgemacht hat. Als Hindernis ist die Schutznormtheorie zu entdecken. Aber eigentlich ist das eine Schutzverhinderungstheorie, und ich meine, die Verfassung der Gemeinschaft sollte in diesem Punkt auch übertragen werden auf den Bereich des Zugangs zum Recht in Deutschland. Dann zu Ihnen, Herr Streinz. Sie haben ein umfassend gestaltetes Referat gehalten, und das findet auch meinen vollen Beifall, aber an einer Stelle habe ich dann doch etwas zu beanstanden. Sie haben so global gesagt, finale Kompetenzen zeichnen das Gemeinschaftsrecht insgesamt aus. Es ist zuzugestehen, dass Art. 94, 95 EG über die Harmonisierung eine solche Kompetenz ist, aber man muss 
gleich hinzufugen, sie ist angebunden an den Binnenmarkt. Man kann noch denken an Art. 308 EG, die Ergänzungsklausel, aber sonst bei den Kompetenzen werden erstens die Aufgaben gefordert, und das ist meines Erachtens ganz wichtig, weil dann der Zweck in dem Rechtsakt enthalten ist, und dann eine präzise Beschreibung, was unter dieser Kompetenz alles getan werden kann. Danke schön.

Classen: Ich möchte zwei Punkte ansprechen: Klagebefugnis und Haftung. Zur Klagebefugnis: Herr Streinz, Sie haben die Möglichkeit angesprochen, bei der Umsetzung von Richtlinien entweder subjektive Rechte auch im deutschen Recht zu schaffen oder nur einen prozessrechtlichen Weg durch Schaffung einer eigenständigen Klagebefugnis zu gehen. Die Frage, in welchen Punkten zwischen den verschiedenen Wegen jeweils praktische Unterschiede bestehen, kann man sicherlich nur differenziert beantworten. Einen Punkt hat Herr Rupp schon angesprochen: Auf den Prüfungsumfang ergeben sich Auswirkungen, ohne dass ich insoweit ein gemeinschaftsrechtliches Problem sehe. In jedem Fall weist der prozessrechtliche Weg den Nachteil auf, dass dann eine auf Art. 19 Abs. 4 GG gestützte Verfassungsbeschwerde nicht mehr möglich ist. Dann stelle ich mir die Frage, ob hier nicht aus Sicht des gemeinschaftsrechtlichen Diskriminierungsverbotes ein Problem besteht, weil aus gemeinschaftsrechtlicher Perspektive die unterschiedlichen Wege, die das deutsche Recht geht, eine Differenzierung wohl nicht rechtfertigen. An Frau Epiney habe ich in diesem Zusammenhang die Frage, ob die konkreten Beispiele, die sie dafür genannt hat, was in Zukunft nach Gemeinschaftsrecht alles eingefordert werden kann, subjektive Rechte begründet (Vorsorgewerte, Schaffung eines Erholungsgebietes), nach ihrer Einschätzung unmittelbar aus den Entscheidungen des EuGH aus dem Jahre 1991 abzuleiten sind, oder ob dies auf eigener Interpretation des Gemeinschaftsrechts beruht. Mit Blick auf das Erholungsgebiet vermag ich nicht zu erkennen, dass dies unter Hinweis auf den EuGH begründet werden kann, und auch bei den Vorsorgewerten habe ich erhebliche Zweifel.

Zum Thema Staatshaftung: Herr Streinz, Sie haben in Ihrer These 30 ausfuhrlich dargelegt, dass jeder vor der Geltendmachung eines Haftungsanspruches Primärrechtsschutz in Anspruch nehmen müsse. Dies setzt voraus, dass dies überhaupt möglich ist. In bestimmten Bereichen gibt es insoweit Probleme. Im Fall von legislativem Unrecht etwa kommt allein eine Verfassungsbeschwerde in Betracht, die aber nur einen erheblich reduzierten Kontrollmaßstab eröffnet. Bei Richtlinien, die auf horizontale Wirkung abzielen, kann zwar ein privatrechtlicher Rechtsstreit im Einzelfall dazu führen, dass das zuständige Gericht ein Umsetzungsdefizit über eine richtlinienkonforme Auslegung ,,in den Griff bekommt“. In an- 
deren Fällen aber ist dies nicht möglich. Von daher muss der in Ihrer These genannte Grundsatz mit Sicherheit in irgendeiner Form eingeschränkt werden. Meine Frage geht dahin, ob man dies so formulieren kann, dass nur der Gebrauch von hinreichend effektiven Rechtsmitteln zu verlangen ist. Dieses Problem ist ja insbesondere auch aus dem Völkerrecht bekannt. Dabei ist insbesondere zu fragen, ob sich der fragliche Rechtsweg bei abstrakter oder auch bei konkreter Betrachtung als effektiv erweisen muss.

Eine zweite Frage zum Haftungsrecht. Sie haben das Problem des Richterprivilegs angesprochen und auf das Problem der Rechtskraft hingewiesen. Ein besonders wichtiges praktisches Beispiel, bei dem man sich eine richterliche Haftung vorstellen kann, ist der Bereich unterlassener Vorlagen trotz entsprechender Vorlagepflicht, wenn dadurch Entscheidungen zustande kommen, die im Widerspruch zur Rechtsprechung des EuGH stehen. Möglicherweise wird diese Konstellation in Deutschland in Zukunft zwar seltener als bislang vorkommen. Im 82. Band war das BVerfG bekanntlich recht zurückhaltend bei der Entwicklung von Fallgruppen, in denen die Verletzung der Vorlagepflicht auch eine Missachtung von Art. $101 \mathrm{GG}$ sein sollte. Jüngst hat jetzt eine Kammer des BVerfG eine Entscheidung des BVerwG aufgehoben und dabei Formulierungen verwendet, die aus meiner Sicht sowohl in der Formulierung als auch in der Sache von dem abweichen, was der Senat als Leitentscheidung vorgegeben hatte. Hier haben wir also einen Fall, dass eine Kammer unter Abweichung von der Rechtsprechung des Senats eine Entscheidung eines obersten Bundesgerichts aufhebt, was es nach einer gestrigen Bemerkung von Herrn Steiner eigentlich nicht geben sollte.

Wenn man aber trotzdem einmal eine unterlassene Vorlage als Beispiel nimmt, so lautet meine Frage, ob eine im Rahmen des Haftungsprozesses erfolgte Vorlage an den EuGH ggf. eine hinreichende Rechtfertigung dafür darstellt, über die Rechtskraft des Vorurteils hinwegzugehen. Wenn also die Rechtskraft im Auge behalten werden muss, dann lautet meine Frage, ob nicht wenigstens eine zwischenzeitlich ergangene EuGH-Entscheidung rechtfertigen kann, die Rechtskraft ein wenig „bei Seite zu schieben“.

Schließlich eine Bemerkung zu Herrn Götz, der sagte, er könne sich vorstellen, dass die Kontrolldichte nationaler Gerichte bei der Kontrolle des Vollzugs von Gemeinschaftsrecht in Zukunft großzügiger sein müsse. Ich bin da nicht ganz so sicher, weil ich nicht ohne Weiteres sehe, dass das Gemeinschaftsrecht hier eine Obergrenze der Kontrolldichte fordern könnte. Für eine Aussage, dass die Gewichte im Verhältnis zwischen Verwaltung und Gerichtsbarkeit in bestimmter Weise zu Gunsten der Verwaltung zu setzen seien, sehe ich im Gemeinschaftsrecht bisher nicht ein- 
mal im Ansatz einen Anknüpfungspunkt. Vielleicht liegt es an meiner begrenzten Phantasie, aber selbst aus dem „effet utile“ vermag ich insoweit nichts abzuleiten. Umgekehrt ist aus meiner Sicht zu fragen, ob nicht das Diskriminierungsverbot in diesem Fall zu folgender Konsequenz führen könnte: Der EuGH schreibt zwar nicht vor, wie dicht die Kontrolle sein muss; Gerichte aber, die im nationalen Bereich relativ intensiv kontrollieren, müssen auch beim Vollzug von Gemeinschaftsrecht ähnliche Kontrollstandards einhalten, so dass im Ergebnis die Deutschen ihr Modell beibehalten müssten. Vielen Dank.

Sommermann: Ich hatte zunächst eine kurze Frage an Herrn Höfling, die bereits von Herrn Zuleeg angesprochen wurde. Wo finden in Ihrem beeindruckenden Entwurf einer umfassenden Rekonstruktion des Rechtsschutzes aus den Grundrechten die Leistungsrechte ihren Platz? Vielleicht haben Sie diesen Aspekt ausgeklammert, indem Sie den Primärrechtsschutz als einen Eingriffsbereinigungsschutz definiert haben. Man könnte mit Ihrem Ansatz natürlich eine Reihe von Verpflichtungsbegehren erfassen, wenn man etwa die Ablehnung eines Genehmigungsantrags als einen Eingriff deutet. Damit erfasst man indes nicht alle Leistungsrechte. Mein nächster Punkt betrifft die Dichotomie zwischen der traditionell Frankreich zugeordneten, in der deutschen Geschichte vor allem von Preußen her bekannten Rechtsschutzkonzeption, in der die Wahrung der objektiven Rechtmäßigkeit des Verwaltungshandelns im Vordergrund steht, einerseits und dem innerhalb der Mitgliedstaaten der Europäischen Union bisher fast ausschließlich Deutschland und Österreich zugeschriebenen Modell eines subjektiven Rechtsschutzes andererseits; zu letzterem wird allenfalls noch Italien genannt, welches bekanntlich einen zweigeteilten Rechtsschutz besitzt, bei dem die Durchsetzung der subjektiven Rechte Sache der ordentlichen Gerichtsbarkeit und die Wahrung der legitimen Interessen Sache der Verwaltungsgerichtsbarkeit ist. Diese Zweiteilung zwischen objektiven und subjektiven Konzepten, die auch Herr Streinz ansprach, lässt sich so bis Mitte der 90er Jahre des 20. Jahrhunderts aufrechterhalten: Frankreich war tatsächlich ein Land, von dem man sagen konnte, dass das objektive Rechtsschutzkonzept überwog. Und das französische Konzept hat bekanntlich das Rechtsschutzverständnis zahlreicher europäischer, etwa auch das der iberischen Staaten geprägt. Seit Mitte der 90er Jahre deutet sich indes in vielen Ländern ein grundlegender Wandel hin zu einer Subjektivierung des Rechtsschutzes an. Dadurch erscheint die bisherige Außenseiterposition unseres Rechts in einem neuen Licht, womit ich nicht sagen will, dass sich Deutschland unter dem Einfluss des durch Rechtsvorstellungen aller Mitgliedstaaten geprägten Gemeinschaftsrechts überhaupt nicht bewegen 
müsse; bisher hat sich jedoch die eng mit dem Rechtsschutzkonzept verbundene gesetzliche Bestimmung über die Klagebefugnis als im Lichte des Gemeinschaftsrechts neu interpretierbar und damit adaptierbar gezeigt. Die Mitte der 90er Jahre einsetzende Subjektivierung des Rechtsschutzes lässt sich deutlich an den iberischen Staaten nachweisen. Nachdem ihre Verfassungen aus den 70er Jahren durch individuelle Rechtsschutzgarantien bereits eine entsprechende Weichenstellung getroffen hatten, erfolgte nunmehr auf einfachgesetzlicher Ebene eine nachholende Modernisierung. Die große Reform des Verwaltungsprozessrechts in Spanien von 1998 ist klar am subjektiven Rechtsschutz ausgerichtet. Man kann dies insbesondere an der Neuregelung des vorläufigen Rechtsschutzes zeigen, da dieser ein typisches Instrument des subjektiven Rechtsschutzes ist, und in Systemen, in denen die Wahrung oder die Wiederherstellung der Integrität der objektiven Rechtsordnung im Mittelpunkt steht, von zweitrangiger Bedeutung ist. Auch der derzeit in Portugal beratene Entwurf einer neuen Verwaltungsprozessordnung betont den subjektiven Rechtsschutz und soll insbesondere den vorläufigen Rechtsschutz stärken. Vielleicht am bemerkenswertesten ist die in Frankreich im Jahre 2000 verabschiedete Reform des Verwaltungsprozessrechts, die am 1. Januar 2001 in Kraft getreten ist. Dadurch werden die Anordnungsrechte des Richters im vorläufigen Rechtsschutzverfahren dem der deutschen Verwaltungsrichter angenähert, mit gewissen Einschränkungen, was die vorläufige Durchsetzung von Leistungsrechten anbetrifft. Die Reform ist im Kontext mit einer neuen Rechtsprechung des Conseil Constitutionnel zu Art. 16 der Erklärung der Menschenund Bürgerrechte von 1789 zu sehen. Während diese Bestimmung früher hauptsächlich als Garantie des Prinzips der Gewaltenteilung in Erscheinung trat, gewinnt sie nunmehr als Garantie eines effektiven Individualrechtsschutzes Bedeutung. Michel Fromont hat in jüngeren Veröffentlichungen diese Subjektivierungstendenz im französischen Verwaltungsprozessrecht herausgearbeitet. Daraus ergibt sich: Man sollte zugunsten einer europäischen Rechtsangleichung nicht voreilig fordern, Deutschland müsse sich in Richtung eines objektiven Rechtsschutzes bewegen; es ist vielmehr auch zu bedenken, dass es zunehmend Bewegungen in die andere Richtung gibt. Vielen Dank.

Rengeling: Ich habe drei Punkte, die ich kurz ansprechen möchte. Der erste Punkt ist folgender. Es ist verschiedentlich gesagt worden, dass das Verfahren dienende Funktion habe und das sei mit dem Gemeinschaftsrecht nicht immer in Einklang zu bringen. Ich würde gerne wissen: Wie ist konkret die Rechtsprechung des Bundesverwaltungsgerichts zu beurteilen, nach der die Außerachtlassung der Öffentlichkeits- 
beteiligung im Hinblick auf $\S 46$ Verwaltungsverfahrensgesetz unschädlich ist? Hält das vor dem EuGH stand? Der zweite Punkt: Wir sagen, und das ist häufig vorgetragen und prognostiziert worden, der Rechtsschutz muss effektiv sein, und es ist eine Systemangleichung gefordert worden und prognostiziert worden, dass wahrscheinlich die Eröffnung der Streitigkeiten großzügiger gehandhabt wird unter dem Einfluss des Europarechts, während die Kontrolldichte zurückgeht. Wie ist das insgesamt eigentlich unter dem Blickwinkel des effektiven Rechtsschutzes zu beurteilen? Ist es für den Kläger letztlich nicht, ich will es mal etwas provozierend sagen, gleichgültig? Was nützt ihm der Zugang zu den Gerichten, wenn nachher in der Sache doch nicht geprüft wird? Also ist das unter dem Blickwinkel des effektiven Rechtsschutzes eigentlich wesentlich, jetzt nicht vom dogmatischen System her, sondern von der Effektivität des Rechtsschutzes aus gesehen? Und die dritte Frage, die ich hätte, ist folgende: Ich glaube auch, dass die Kontrolldichte unter dem Einfluss des Europarechts abnehmen wird. Ich möchte einen Punkt anschneiden, zu dem vielleicht etwas gesagt werden kann. Wir haben seit kurzem das so genannte Artikelgesetz und darin die Anforderungen des integrierten Umweltschutzes konkretisiert oder besser gesagt umgesetzt, konkretisiert werden müssen sie noch. Die Tendenz in der Bundesrepublik ist stark, das bisherige System beizubehalten, nämlich das System, durch untergesetzliche Regelwerke unbestimmte Gesetzesbegriffe zu konkretisieren, um Rechtssicherheit zu haben. Nun muss die Umwelt insgesamt geschützt werden. Keiner weiß so richtig, was das ist, aber ist da nicht schon vorgegeben, dass hier eine Entscheidung zu treffen ist, die so schwierig gerichtlich überprüfbar sein wird, dass auch insofern das europäische Recht zwangsläufig dazu führen wird, die Kontrolldichte zu begrenzen? Vielen Dank.

Iliopoulos-Strangas: Ich möchte eine ergänzende Bemerkung zur These Nr. 6 des eindrucksvollen Referats von Herrn Streinz machen. Grundsätzlich stimme ich mit dem Referenten darin überein, dass Art. 41 EMRK eine dritte Säule des europäischen Staatshaftungsrechts und damit des sekundären Rechtsschutzes begründet. Eine gewisse Relativierung erscheint jedoch angezeigt. Ein endgültiges Urteil des Europäischen Gerichtshofs für Menschenrechte, das der verletzten Partei gemäß Art. 41 EMRK eine gerechte Entschädigung zubilligt, stellt nämlich nach weit überwiegender Meinung im Schrifttum für die verletzte Partei keinen vollstreckbaren Titel dar. In der Tat fehlt in der EMRK eine den Art. 244 und 256 EG vergleichbare Regelung, wonach die Urteile des EuGH vollstreckbare Titel sind und ihre Zwangsvollstreckung nach den Vorschriften des Zivilprozessrechts des Staates erfolgt, in dessen Hoheits- 
gebiet diese Zwangsvollstreckung stattfindet. Auch kommt eine entsprechende Ergänzung der EMRK nicht in Betracht, weil es Vertragsstaaten gibt, die - dem Dualismus folgend - die EMRK nicht zum Bestandteil ihrer nationalen Rechtsordnung gemacht haben und machen wollen. So stellt nach einhelliger Auffassung im Schrifttum die in Art. 46 Abs. 1 EMRK festgelegte Verpflichtung der Vertragsparteien, die sie betreffenden endgültigen Urteile des Gerichtshofs zu befolgen, eine rein völkerrechtliche Verpflichtung dar, deren Nichterfüllung durch verurteilte Staaten lediglich deren völkerrechtliche Haftung nach sich zieht. Anders verhält es sich allerdings, wo das jeweilige nationale Recht von sich aus die innerstaatliche Vollstreckbarkeit solcher EGMR-Urteile eingeführt hat. Das ist aber nur in ganz wenigen Vertragsstaaten geschehen, zu denen etwa Malta gehört. Überdies häufen sich die Fälle, in denen verurteilte Staaten sich weigern, die vom EGMR zugebilligte Entschädigung zu zahlen. Somit kann es dazu kommen, dass ein Bürger zwar mit seiner Individualbeschwerde in StraBburg erfolgreich ist und durch EGMR-Urteil eine gerechte Entschädigung zugesprochen erhält, die Vollstreckung dieses Urteils aber nicht mit rechtlichen Mitteln durchsetzen kann, sondern auf die - durch ihn nicht erzwingbare - Bereitschaft des Vertragsstaates zur Erfüllung seiner völkerrechtlichen Befolgungsverpflichtung aus Art. 46 EMRK angewiesen bleibt. Wo es daher keine ausdrückliche gesetzliche Regelung über die innerstaatliche Vollstreckbarkeit von EGMRUrteilen gibt (und, soweit ersichtlich, gibt es eine solche in Deutschland nicht), ist die Effektivität des Sekundärrechtsschutzes über Art. 41 EMRK rechtlich nicht gesichert.

Vorsitzender: Schönen Dank, Frau Iliopoulos-Strangas. Am Ende unserer Aussprache haben die Referenten Gelegenheit zu einem ausfuihrlichen Schlusswort, und zwar - wie üblich - in der gegenüber den Referaten umgekehrten Reihenfolge. Dementsprechend darf ich zuallererst Frau Epiney das Wort erteilen.

Epiney: Ich möchte zu drei Punkten Stellung nehmen, die in verschiedenen Variationen in den Wortbeiträgen angesprochen wurden. Zunächst der erste Punkt: Ich möchte meinen Begriff der normativen Interessentenklage in Erinnerung rufen bzw. präzisieren: Mir ging es hier darum, insofern einen Gegensatz zu der Rechtslage in Frankreich und auch der Schweiz herzustellen, dass es im Gemeinschaftsrecht eben gerade nicht um die Betroffenheit bzw. ein Interesse an der Aufhebung einer Entscheidung geht, sondern darum, dass die geltend gemachte Rechtsnorm das Interesse des Einzelnen schützen muss und insofern eine gewisse Parallelität zu der Rechtslage in Deutschland besteht. In diesem Zusammenhang ist es 
auch noch interessant, dass ein Richter des Europäischen Gerichtshofs (es war nicht Herr Zuleeg) betont hat, der EuGH habe das deutsche Schutznormkonzept übernommen. Dies war sicherlich nicht so gemeint, dass der Inhalt übernommen wurde, aber vielleicht in dem Sinn, dass der dogmatische Ansatzpunkt aufgegriffen wurde. Der zweite Punkt, den ich hier erwähnen möchte, betrifft die Frage des Rechtswidrigkeitszusammenhangs. Es ist ja so, dass dem Gemeinschaftsrecht nur entnommen werden kann, dass die jeweiligen individuellen Rechte von den Betroffenen auch geltend gemacht werden können müssen. Dies impliziert dann aber auch, dass ein Rechtswidrigkeitszusammenhang in dem Sinn, dass das zuständige Gericht nur die Verletzung dieser Rechte prüft, zulässig ist. Eine objektive Rechtmäßigkeitskontrolle würde über die (zwingende) Reichweite der individuellen Rechte des Gemeinschaftsrechts hinausgehen, ist aber natürlich möglich. Darüber hinaus muss der Rechtsschutz effektiv sein, d.h. also insoweit die individuellen Rechte geltend gemacht werden können, muss entsprechend $\S 113$ vielleicht nicht zwingend angepasst, aber jedenfalls gemeinschaftsrechtskonform ausgelegt werden. Damit komme ich auch in diesem Zusammenhang zu der Frage der durch das Gemeinschaftsrecht vorgesehenen Folgen der Verletzung von Bestimmungen über Öffentlichkeitsbeteiligung, die Frage von Herrn Rengeling. Effektiver Rechtsschutz heisst tatsächlich, dass in dem Fall, in dem die Rechte geltend gemacht werden, auch eine effektive Sanktion erfolgen muss. Es gibt hier Zitate aus der Rechtsprechung des Europäischen Gerichtshofs in Bezug auf die Folgen der Verletzung von Bestimmungen über die Öffentlichkeitsbeteiligung, die nach meiner Erinnerung auf die UVP-Richtlinie Bezug nehmen. In der Sache spricht sich der EuGH dort dafir aus, dass die Geltendmachung einer Verletzung dieser Rechte wirksam sein muss, und dann ist die logische Schlussfolgerung hieraus - und auch eine neuere Entscheidung des Bundesverwaltungsgerichts dürfte in diese Richtung gehen natürlich schon, dass eine Verletzung der Beteiligungsrechte nicht allgemein ohne Folgen für die Rechtmäßigkeit der Verwaltungsentscheidung bleiben kann, so dass man nicht anführen kann, die Verletzung der Verfahrensrechte sei nicht so schlimm gewesen und die Entscheidung hätte so oder so gleich getroffen werden müssen. Eine andere Frage ist, ob bei verschiedenen Arten von Beteiligungsrechten nicht noch eine Differenzierung notwendig ist. Jedenfalls aber bei den im Umweltrecht typischen Beteiligungsrechten ist die Folge ihrer Verletzung grundsätzlich die Rechtswidrigkeit der jeweiligen Verwaltungsentscheidung. Damit komme ich zum dritten Punkt: die Frage des Strukturwandels bzw. Systemwandels. Selbstverständlich ist es so, dass die Ausgestaltung der Klagebefugnis in das jeweilige Verwaltungsrechtssystem einzubetten ist, wobei ich dies in der Kürze der Zeit nicht leisten konnte, die hier in erster Linie wichtigen 
Aspekte aber im Wesentlichen bekannt sein dürten. Ich glaube aber nicht, dass die Gemeinschaft, wie es ja zumindest in der Diskussion teilweise vertreten wurde, das französische System übernommen hat. Vielmehr geht es hier - wie bereits im Vortrag erwähnt - wirklich um eine Art Verschmelzung verschiedener Traditionen. In diesem Zusammenhang ist sodann zu erwähnen, dass auch das deutsche Recht sowohl in Bezug auf die Klagebefugnis als auch in Bezug auf die Kontrolldichte nicht unflexibel ist, sondern dass hier in den vergangenen Jahren oder Jahrzehnten doch einige Entwicklungen zu beobachten sind. Man denke nur an die Anfechtung von Risikoanlagen oder an die Rechtsprechung zur normativen Ermächtigung bei der Zurücknahme der Kontrolldichte der Gerichte. Dabei beruht diese Rechtsprechung nicht auf zwingenden Vorgaben des Gemeinschaftsrechts. Vor diesem Hintergrund sollten wir wohl vorsichtig sein, jetzt sofort von einem grundlegenden Systemwandel zu sprechen. Ich glaube eher an eine Weiterentwicklung des geltenden Systems hin zu einer erweiterten Klagebefugnis, aber auch hin zu einer gewissen Zurücknahme der Kontrolldichte. Ich habe nicht den Eindruck, dass eine gewisse, „kontrollierte“ Zurücknahme der Kontrolldichte dann zwangsläufig zur Ineffektivität des Rechtsschutzes führen muss, zumindest nicht im Vergleich zur jetzigen Rechtslage mit ihrer langen Verfahrensdauer. Dies ändert freilich nichts daran, dass es sinnvoll sein dürfte, die Zurücknahme der gerichtlichen Kontrolle normativ zu regeln. Ein m.E. gelungenes Beispiel haben wir ja im UGB-Entwurf formuliert. Die Frage, ob es nicht deshalb einen Rückgang der Effektivität gerichtlichen Rechtsschutzes geben wird, weil im Gemeinschaftsrecht sehr viele unbestimmte Rechtsbegriffe verwendet werden, ist berechtigt. M.E. ist dies aber keine zwangsläufige Entwicklung, sondern es kommt wesentlich auf die Umsetzung an. So ist etwa der Begriff des ,integrierten Umweltschutzes“ bzw. der „Schutz der Umwelt als Ganzes" sicherlich ein sehr diffuses Konzept, das ich im Übrigen auch nicht nur positiv einschätze. Es ist auch schwer vorstellbar, dass man solche Verpflichtungen in irgendeiner Form einklagt, da schlicht und ergreifend die Bestimmtheit fehlt. Daher ist hier der Umsetzungsgesetzgeber gefordert, der dann den ihm in den einschlägigen Rechtsakten eröffneten Spielraum nutzen und hier präzisere Handlungsvorgaben erlassen kann. Jedenfalls kommt eine Pflicht der Mitgliedstaaten, Einzelnen gerichtlich durchsetzbare Rechte einzuräumen, nur dann in Betracht, wenn diese (im Umsetzungsrechtsakt) hinreichend bestimmt sein müssen. Spielräume bei der Umsetzung hin zu präziseren Vorgaben bestehen aber in aller Regel. Danke schön.

Streinz: Zunächst möchte ich mich beim Vorstand und den Mitreferenten bedanken, dass ich das Thema so behandeln durfte und dabei man- 
ches gelesen habe, was mir bisher fahrlässigerweise entgangen ist. Dank auch für die vielen Diskussionsbeiträge. Ich möchte antworten, soweit ich direkt angesprochen worden bin oder indirekt, und soweit mir hier ein Wahrnehmungsmangel unterläuft, bitte ich dafür um Verzeihung. Zunächst zu Ihnen, Herr Schoch. Die Asymmetrie des Rechtsschutzes durch die Privilegierung der Eilverfahren, was zu einer Steigerung dieser Verfahren führt, ist ein interessantes Phänomen. Wenn ich das richtig gesehen habe, gibt es dazu in den Niederlanden bereits eine starke Tendenz. Es stellt sich die Frage, ob dies gewünscht wird, da die Verfahren schnell erledigt werden, oder ob dies eine unbeabsichtigte Nebenfolge ist. Differenzierte Regelungen sind in der Tat problematisch, zum einen für den Rechtsanwender, aber auch hinsichtlich der Rechtfertigung differenzierter Maßstäbe. Hier besteht ein Harmonisierungsdruck im Sinne eines Rechtfertigungszwangs für unterschiedliche Regelungen, zum Teil rechtlich, zum Teil tatsächlich. Ich möchte aber nicht generell ausschließen, dass unterschiedliche Regelungen auch ihren Sinn haben können. Ich möchte das so verstanden wissen, dass man prüfen soll, ob man unter Umständen etwas, was man gemeinschaftsrechtlich, in Gemeinschaftsrechtsfallen, nicht halten kann, aus unterschiedlichen Erwägungen für rein nationale Fälle noch bewahren soll. Die Frage Bewahrung oder Veränderung ist natürlich dann im Sinne letzterer zu beantworten, wenn ohnehin erkannte Defizite mitbeseitigt werden können. Über das Ausmaß der Defizite bestehen aber zum Teil unterschiedliche Auffassungen. Zum Kohärenzgebot: Meiner Ansicht nach hat der EuGH das Erfordernis der Kohärenz mehr behauptet als begründet, und zwar deshalb, weil er keine Mängel bei der Anwendung von Äquivalenzgrundsatz und Effizienzgebot, was ja zuvor offenbar genügt hat, dargetan hat. Ich kann es nur so erklären, dass der EuGH vielleicht meinte, wie ich es auch im Vortrag schon versuchte anzudeuten, dass in den Fällen, in denen es um die vorübergehende Nichtanwendung von Gemeinschaftsrecht geht, es sich eigentlich um eine Kompetenz des EuGH handelt und daher auch die für ihn vorgeschriebenen Maßstäbe gelten sollten, und er davon ausgegangen ist, dass die Regelungen in Art. 242, 243 EG-Vertrag Ausdruck eines allgemeinen Rechtsgrundsatzes sind. Ob dies der Fall ist, ist allerdings fraglich. Vor allem hat der EuGH auch noch das Urteil Factortame einbezogen, und da greift dieser Ansatz nicht. Entscheidend ist aus meiner Sicht, wie man damit umgeht, d.h., was es konkret bedeutet, das Gemeinschaftsinteresse in angemessenem Umfang zu berücksichtigen. Hier wird die Gefahr gesehen, dass der EuGH ein zu großes Gewicht darauf legt und keine richtige $A b w a ̈ g u n g ~ v o r n i m m t . ~ O b$ das so ist, kann man jetzt wohl noch nicht abschließend beurteilen, aber auf diesen Punkt werden wir sicher besonders achten müssen. Es ist eine Strukturentscheidung, 
was sie, Herr Breuer, angesprochen haben. Die Frage ist: Brauchen wir dafür den parlamentarischen Gesetzgeber? Inwieweit trägt der Rahmen, den wir parlamentarisch bereits legitimiert haben, dies noch, oder wann überschreiten wir ihn? Wenn man das Staatshaftungsrecht gesetzlich normiert, würde ich, wie in anderen Fällen auch, empfehlen, dass man beim Überdenken des Konzepts die seitherige Entwicklung auf internationaler und europäischer Ebene einbezieht, was man ja auch bei der Zivilrechtsreform und anderen Reformen getan hat oder jedenfalls tun sollte. Dabei kann man natürlich auch auf wissenschaftlichen Erkenntnissen aufbauen, was Herr Maurer hervorgehoben hat. Zu Herrn Klein: Ihre Ausführungen zur Instrumentalisierung finden meine volle Zustimmung. Denn subjektive Rechtsverfolgung und Instrumentalisierung schließen sich nicht aus. Man sollte dem Begriff Instrumentalisierung den negativen Touch nehmen, der zum Teil natürlich auch daher kommt, dass es nicht fein ist, einen Konkurrenten bei der Kommission anzuschwärzen. Aber das Recht kann oft nur so durchgesetzt werden, und dann ist das Einschreiten der Kommission auch eine Art Prämie für den einzelnen, der sich um das Recht kümmert, eigentlich auch irgendwie ein marktwirtschaftlicher Aspekt. Auch ich sehe „Francovich“ nicht als so entscheidenden weiteren Schritt wie das Urteil zur unmittelbaren Wirkung, sondern eigentlich nur als eine Fortentwicklung desselben. Zu meiner These 25: Dass das Gebiet effektiven Rechtsschutzes nicht eindimensional verstanden werden soll, möchte ich nicht als Beschränkung verstanden wissen, sondern es bezieht sich darauf, dass die verschiedenen Funktionen gesehen werden sollen, dass man also nicht nur die Effektivität des Gemeinschaftsrechts als solche völlig losgelöst von den Werten sieht, die das Gemeinschaftsrecht selbst enthält, insbesondere die subjektiven Rechte, und die Weiterung zu einem Dreiecksverhältnis erkennt, da das Gemeinschaftsrecht als solches auch im gemeinsamen Interesse der Mitgliedstaaten liegt, was bei der Auslegung von Vorschriften ggf. mitberücksichtigt werden kann und vom EuGH auch, wenn entsprechend vorgetragen, oft mitberücksichtigt wird. $\mathrm{Zu}$ Herrn Maurers Frage nach dem primären und sekundären Rechtsschutz und wie das Francovich-Urteil umgesetzt werden soll. In der Tat gibt es hier die beiden Möglichkeiten. Einmal den Weg, den der Bundesgerichtshof gewählt hat, das Trennungsmodell, und zum anderen das Verbindungsmodell. Die Wahl des Letzteren liegt nahe, wenn man das deutsche Staatshaftungsrecht für defizitär hält, was ja verbreitete Ansicht ist, wenn auch viele, die es für defizitär halten, es dennoch ganz gut bewältigen, wie Sie sagten. Dann könnte man diese Defizite bei einer vereinheitlichten Lösung abbauen. Man müsste nur bedenken, ob hier neue Probleme oder Brüche entstehen könnten, aber das ist eine Frage, die man sich ja ohnehin bei jeder Reform stellen muss. Zu Herrn Schachtschnei- 
der: In der Tat gibt es eine Reihe von Fällen, in denen eine europäische Rechtsentwicklung positive Anstöße gibt. Dass in großen politischen Fragen der Rechtsschutz reduziert ist, hängt zusammen mit dem Wesen einer politischen Entscheidung. In diesem Fall besteht der „Rechtsschutz“ darin, dass man die Verantwortlichen abwählt. In der Tat: Je weiter die Klagemöglichkeit ist, um so reduzierter ist die Kontrolldichte, und das ist auch das, was Herr Rengeling angesprochen hat. Es stellt sich irgendwann die Frage: Ich kann zwar gegen alles klagen, ich werde an allem beteiligt, aber es ändert sich nichts. Das ist sicherlich auch keine befriedigende Lösung, und in diesem Sinne sollte ich mit meiner Forderung nach Ausbalancierung verstanden werden. Zum Problem Verwerfungsmonopol des Bundesverfassungsgerichts durch Art. 100 Abs. 1 GG und der Verwerfungspflicht von Gerichten, auch schon von Verwaltungsbeamten. Das ist durchaus ein Problem, aber es ist aus meiner Sicht eine zwingende Folge des Anwendungsvorrangs des Gemeinschaftsrechts. Man hat ja überlegt, ob man Instanzen vorschalten sollte. Das haben einige Staaten auch getan und der EuGH hat entschieden, das gehe nicht, und der Grund dafür ist, weil die Klärung durch den EuGH verzögert wird. Das Problem ist der Verzögerungseffekt. Es wird während dieser Zeit das Gemeinschaftsrecht nicht angewandt und daher fordert der Vorrang des Gemeinschaftsrechts, dass es unverzüglich, unter Außerachtlassung entgegenstehenden nationalen Rechts, angewandt werden muss. Interessant fand ich gerade auch aus Sicht meiner interdisziplinären Zusammenarbeit im Rahmen unserer Bayreuther Rechts- und Wirtschaftswissenschaftlichen Fakultät die Folgerungen aus der ökonomischen Analyse des Rechts, die Herr Engel gezogen hat. Diese Anreizfunktion trägt sicherlich auch sehr stark zur Effektivität des EG-Rechts bei. Nur ein Beispiel: Nach der MP-Travel-LineGeschichte wird man es sich doch sehr überlegen, ob man noch öfter die Umsetzung von Richtlinien ohne weiteres verzögert, oder ob das nicht auf Dauer zu teuer wird. Die Frage ist: Wer haftet dafür? Nur der Steuerzahler? Aber man muss auch einräumen, dass es den Beamten, und das hat Herr Meyer angesprochen, nicht gleichgültig ist, wenn hier Rechtsfehler gemacht werden. Zu Herrn Spannowsky: Der Vorrang des Primärrechtsschutzes ist gemeinschaftsrechtlich geboten, soweit der Verstoß an der Quelle beseitigt werden kann, also ist in der Tat die unmittelbare Wirkung von Richtlinien vor „Francovich“ zu prüfen. Der konkrete Fall wäre, wenn das in diese Richtung geht, auch so zu lösen. Ihr Beispiel zeigt auch wieder, dass iura novit curia manchmal doch noch eine widerlegliche Vermutung im Gemeinschaftsrecht ist, was aber auch in diesem Gebiet liegt, an seiner Unüberschaubarkeit. Man muss wirklich, auch wenn man meint, sich auf einem Gebiet gut auszukennen, in jedem Einzelfall erforschen, ob es im EG-Recht etwas (Neues) gibt. Wir haben ja jetzt mo- 
derne Hilfsmittel, die hoffentlich auch den Behörden zur Verfügung stehen werden. Und insoweit besteht auch wohl eine Sicherstellungspflicht der Mitgliedstaaten, dass ihre Behörden das Gemeinschaftsrecht kennen. Noch einmal zu Herrn Meyer. Die Frage nach dem Begriff „Primär- und Sekundärrechtsschutz" habe ich mir auch gestellt. Denn der Begriff war ja nicht unbestritten, hat sich aber durchgesetzt. Ich glaube, das Problem liegt darin, dass man manchmal den sekundären Rechtsschutz als „sekundär" ansieht. Das soll er ja nicht sein im Sinne einer Wertigkeit. Auch er ist eine Form des Rechtsschutzes, so dass der Begriff nicht mit einer Wertigkeitsvorstellung, sondern mit dem Vorrang des Primärrechtsschutzes, der aus den genannten Gründen der Abwendung der Rechtsverletzung an der Quelle geboten ist, verbunden werden sollte. Herr Rauschning, vielen Dank für den Hinweis zu dieser völkerrechtlichen Frage, die ich mit einbeziehen kann. Herr Götz, meine Schlussthese mit „Bewahrung und Veränderung" von Strukturen knüpfte natürlich an das Thema der Tagung von 1964 an. Ich meine, dass diese maßvollen schonenden Elemente auch im Gemeinschaftsrecht selbst angelegt sind, man muss sie nur sehen und sehen wollen, gerade auch in der Rechtsprechung des Europäischen Gerichtshofs. Sehr interessant war Ihr Beispiel aus dem Arzneimittelrecht. Wenn man einen gemeinschaftsrechtlich einheitlich geregelten Bereich durch eine Arzneimittelrichtlinie hat, so hat man auch einheitliche Auslegungskriterien, jedenfalls soweit die Harmonisierungsdichte reicht. In den seltensten Fällen wird hier noch ein darüber hinausgehendes nationales Ermessen gegeben sein. Allerdings, wenn ich das an dieser Stelle sagen darf, nehmen in letzter Zeit die Urteile des Europäischen Gerichtshofs zu, die die Beurteilung der Einzelfälle ausdrücklich den nationalen Gerichten überlassen, was an sich richtig ist, aber nicht ohne gemeinschaftsrechtliche Vorgabe erfolgen darf, so dass über die Entscheidungsfreiheit der nationalen Gerichte Unsicherheit besteht. Herr Zuleeg, die finale Struktur ist mit Sicherheit kein Freibrief für Kompetenzüberschreitungen, da wäre ich ganz missverstanden worden. Sie haben völlig zu Recht den Binnenmarktbezug von Art. 94, 95 EG-Vertrag hervorgehoben, was der EuGH auch im Tabakwerbeverbotsurteil getan hat. Was Sie sagten, Herr Sommermann, bestätigt die wechselseitigen Einflüsse und auch die Relativität von Typisierung, vor allem von älteren Typisierungen, die immer wieder nachgetragen werden müssen. Herr Classen, die Lösung über $\S 42 \mathrm{Abs}$. 2 VwGO und Art. 19 Abs. 4 GG habe ich hinsichtlich der gemeinschaftsrechtlichen Zulässigkeit in einer Fußnote angesprochen, der Text ist der Zeit zum Opfer gefallen. Meiner Meinung nach, aber das wäre noch zu überdenken, genügt es dem Äquivalenzgrundsatz. Denn im deutschen Recht ist diese Lösung allgemein vorgesehen, und die gemeinschaftsrechtlichen materiellen Vorgaben können 
erfüllt werden. Anders sieht es allerdings aus, wenn man das sog. Diskriminierungsverbot soweit versteht, dass eine Maximallösung jedenfalls angestrebt werden muss. Da ist einiges noch umstritten. Zur These 30: Hier ist sicherlich folgende Einschränkung hinsichtlich der Zumutbarkeit zu machen: Es dürfen Unsicherheiten hier nicht zu Lasten des Klägers gehen. Dies war übrigens auch ein Problem im Brasserie-Fall mit der Frage, was für eine Klage denn erhoben werden hätte sollen. Dies ist letztlich nicht zum Tragen gekommen, weil der BGH die Klage aus anderen Gründen abgewiesen hat. Bei judikativem Unrecht kommt vor allem wohl ein möglicher Verzögerungsschaden im Betracht. Zu Herrn Rengelings Frage zu § 46 VwVfG. Wenn das Verfahrensrecht als solches vom Gemeinschaftsrecht als subjektiv klagbares Recht eingeräumt ist, dann muss das gewährleistet werden, und dann kann $\S 46 \mathrm{VwVfG}$ das nicht aushebeln. Es kommt darauf an, wie weit die Dichte der gemeinschaftsrechtlichen Vorgabe ist. Bei der Balance, beim Ausbalancieren von Klagerechten und Kontrolldichte, ist in der Tat eine Gewinn- und Verlustbilanz aufzustellen. Denn das eine geht nicht ohne Rücksicht auf das andere, und da stellt sich die Frage, was für den Bürger wirklich von Vorteil ist. Die Kontrolldichte wird in manchen Bereichen abnehmen, aber das ist nichts Ungewöhnliches, denn wir typisieren bei der Kontrolldichte ja auch im deutschen Recht nach verschiedenen Sachbereichen und Sachverhalten. Zuletzt zu Ihnen, Frau Iliopoulos-Strangas. Vielen Dank für die wichtigen Hinweise zu Art. 41 EMRK, die auch zeigen, dass völkerrechtliche Institute auf andere Weise realisiert werden müssen. Wenn man freilich bedenkt, welche schwachen Sanktionsmöglichkeiten das Völkerrecht in vielen Bereichen hat, ist die Effektivität doch ganz beachtlich. Es gibt zum Teil Vollzugsprobleme bei Entscheidungen des EGMR. Wenn ich das richtig in Erinnerung habe, ist wegen Nichtbefolgung des Matthews-Urteils jetzt eine Mahnung erfolgt. Eine andere Lösung wäre, dass auch ohne Verpflichtung aus der EMRK das innerstaatliche Recht entsprechende Ansprüche klagbar stellt. Vielen Dank.

Höfling: Vielen Dank, Herr Bauer. Mir fehlt die Systematisierungskraft, das jetzt nach Sachgesichtspunkten wirklich zu strukturieren. Ich werde deshalb der Reihe nach versuchen, zu antworten. Zunächst zu Herrn Schoch, der weitgehend zugestimmt hat, was ja einfach ist, weil ich mich im Wesentlichen auf seine wegweisenden Vorarbeiten beziehen konnte. Was ich andeuten wollte mit diesem binnenpluralistischen Gerichtsschutzmodell, Herr Spannowsky hat es ja auch gesagt: Für den Bereich der sog. sekundären Unrechtshaftung glaube ich, dass vieles für eine Konzentration bei den öffentlich-rechtlichen Gerichten, also bei den Verwaltungsgerichten spricht. Dogmatisch sowieso, wenn man es so begrün- 
det, wie ich es versucht habe. Ich habe nur davor gewarnt, weiter gehend zu sagen, wir müssen auf dem Weg fortführen und das monopolisieren. Sie haben selbst eben auf ein Beispiel, das ich auch genannt habe, hingewiesen. Wo wären wir und ständen wir bei der Abwehr kommunaler oder staatlicher Wirtschaftstätigkeit, hätten wir nicht die wettbewerbsrechtlichen Interventionen der Zivilgerichte. Das Wettbewerbsrecht überhaupt, das gilt für einen weiten Teil deregulierter Gebiete, spielt eine ganz große Rolle und müsste weiter entwickelt werden als flankierender Rechtsschutz in diesen Gebieten. Der ganze Bereich der Selbstverpflichtungen im Umweltrecht und im Wirtschaftsrecht: Drittschutz ist z.T. nur zu erreichen über eine stärkere Aktivierung des Wettbewerbsrechts; das träte ergänzend hinzu und würde zusätzlich Kontrolldichte bringen, die wir brauchen. Man könnte weiterhin meinen, selbst ein Institut wie der Amtshaftungsanspruch bringt uns noch was, wenn wir die hochproblematische Praxis der Rechtskontrolle von personalwirtschaftlichen Entscheidungen im öffentlichen Dienst betrachten. Wenn wir da nicht wenigstens den Amtshaftungsanspruch hätten, bliebe alles ungerügt, was dort an rechtswidrigen Besetzungen und zwar unter Verstoß gegen eine grundrechtsgleiche Garantie, nämlich Art. 33 Abs. 2, passiert. Dass wir jetzt den Vergaberechtsschutz bei den Zivilgerichten haben, hat ja auch etwas damit zu tun, dass offenkundig die Verwaltungsgerichte nicht in der Lage sind, Rechtsschutz in angemessener Zeit zu gewähren. Ich glaube, wir kommen schon in diesen Gebieten nicht aus ohne die Zivilgerichte, und wir werden erst recht nicht auskommen in diesen Auffangordnungen, die wir in Zukunft haben. Deshalb war das mein Plädoyer, und das schließt an, an das, was Herr Schuppert gesagt hat; dem kann ich nur zustimmen. Nur weiß ich nicht, ob für alle Bereiche ein Verwaltungskooperationsrecht ausreichend wäre; im gerade eben angesprochenen Bereich der Selbstverpflichtungen, da glaube ich müssen wir auch auf andere Instrumente setzen. Herr Breuer, wenn ich ihn recht verstanden habe, hat versucht, das System des sog. Sekundärrechtsschutzes ein wenig in Schutz zu nehmen im Blick auf die in der Tat beeindruckenden Darstellungen unserer junggebliebenen Altmeister des Staatshaftungsrechts. Im Grunde genommen geht dies aber nur, weil aus didaktischen Gründen diese Darstellungen dogmengeschichtlich und rechtsgenetisch orientiert sind. Damit aber, so müsste man eigentlich sagen, wirken sie systemstabilisierend für ein Unsystem, das eigentlich so nicht existiert, wie es dort dargeboten wird. Zu Herrn Sachs. Keine Frage, mir fehlte die Zeit, diese grundrechtsdogmatischen Grundsatzfragen so angemessen zu reflektieren, wie das Ihnen angemessen wäre. Zwei Dinge dennoch: Ich habe wenig Bedenken mit dem, was ich in These $8 \mathrm{~b}$ angesprochen habe. Ich glaube schon, dass man den restitutorischen Beseitigungsanspruch als unmittelbaren ab- 
wehrrechtlichen Anspruch konstruieren kann. Es bleibt bei der Rückbeziehung auf das Schutzgut, dessen Integrität zweifelsohne im Zentrum des Abwehrrechts steht. Und ich halte es für schlechterdings, Herr Schoch hat dafür die nötigen deutlichen Worte gefunden, unvertretbar, dass der VGH in Baden-Württemberg sagt, die Mauer, die kannst du nicht wieder aufrichten lassen, die alten Steine sind nicht mehr da. Dass diese Art von Verneinung eines Restitutionsanspruchs mit Grundrechtsdogmatik nun gar nichts mehr zu tun hat und der Vorgänger dieses Gerichts schon vor 50 Jahren zu einem anderen Ergebnis gekommen ist ohne entwickelte Grundrechtsdogmatik, das sollte doch zu denken geben. Was die These $8 \mathrm{Nr}$. c angeht, da hatte ich eigentlich gedacht, ich sei eher in Ihren Gewässern gewesen, das als grundrechtsmittelbaren Berechtigungskomplex anzuerkennen. Vom Gesetzgebungsauftrag haben Sie gesprochen, ich habe versucht zu formulieren, es ist sozusagen auch ein Zwischenwesen: Der Gesetzgeber ist verfassungsrechtlich gezwungen, so was zu machen, aber es muss in einfaches Recht gegossen werden. Und wenn dieses einfache Gesetz oder dieses einfache Recht durch den Gesetzgeber nicht kommt, dann haben es die Gerichte zu machen. Von Herrn Maurer war weitgehend Zustimmung zu hören, ich kann nur noch mal unterstreichen, man sollte den Art. 34 in der Tat ernst nehmen. Er hat zu Recht auch auf einige Vorgänger und literarische Stimmen hingewiesen, die noch mal deutlich machen, dass wir es mit einem jubiläumsträchtigen Thema zu tun haben. Der 9. Deutsche Juristentag hat schon 1871 dafür plädiert, eine unmittelbare Staatshaftung einzuführen, das was man mit Art. 34 machen könnte und müsste heute. Er hat zu Recht auch auf Bachofhingewiesen, auch das ist ein Jubiläum, vor 50 Jahren ist die bahnbrechende Arbeit zum Folgenbeseitigungsanspruch erschienen und vielleicht nicht ganz so bahnbrechend, immerhin aber auch 20 Jahre alt, ist die Nassauskiesungsentscheidung. So ist es in der Tat ein sehr jubiläumsträchtiges Thema gewesen. Herrn Engel kann ich nur für die originelle Unterstützung der Konzeption danken, dem ist nichts hinzuzufugen. Ja, Herr Püttner hat den Starrsinn des BGH angesprochen, aber zu bedenken gegeben, ob wir nicht doch auf den Gesetzgeber warten müssen; ich habe keine Hoffnung, und ich weiß auch nicht ganz, ob es nur daran liegt, dass sich Bund und Länder nicht einigen können. Die Justizministerin hat im Moment, glaube ich, so viele Jahrhundertprojekte, dass das mit dem Staatshaftungsrecht einfach aus diesen Gründen noch etwas auf sich warten lässt. Und dann gibt es eben die Möglichkeit bei der Konstruktion der Staatsunrechtshaftung, dass das, was der BGH nicht leistet, dann eben die Verwaltungsgerichte leisten und nicht unbedingt auf den Gesetzgeber warten müssen.

Nun zu den letzten Beiträgen, die mich ja sozusagen in die Zange ge- 
nommen haben, wenn ich das recht verstanden habe. Herr Zuleeg und auch Herr Sommermann haben gesagt, das war zu wenig; und die Konstitutionalisierungsgegner mit der geballten Kraft in der Person von Herrn Meyer haben gesagt, das ist alles viel zu viel. Um bei den ersteren anzufangen: Ich habe betont, dass es sich um einen skizzenhaften Bauplan handelt. Das schließt nicht aus, dass man auch darüber nachdenkt, ob Leistungsrechte, wenn sie definitive Ansprüche vermitteln, in dieses System zu integrieren wären und einfachrechtliche subjektiv-rechtliche Rechte ebenfalls, wenn diesen auch ein materialer Reaktionsanspruch entnommen werden kann. Das wäre zu leisten, darüber müsste man genauer nachdenken. Wie gesagt, dazu war ich dann nicht mehr imstande. $\mathrm{Zu}$ Herrn Meyer und Herrn Wahl und Herrn Pietzcker und Herrn Götz, wenn ich das recht verstanden habe, also erstens: Sie haben gesagt, das sei ein Fehler, das alles nach demselben Muster zu machen; das Gegenteil darzulegen habe ich versucht. Ich glaube auch nicht, dass es terminologisch schädlich ist, von Rechtsschutz zu sprechen. Es geht um den Schutz des Rechts, ob man den sekundär nennt oder nicht, es ist Rechtsschutz. Das eigentliche Problem, da wo Sie offenkundig die großen Bauchschmerzen haben: Steht das in der Verfassung drin. Das hat nun schon ein bisschen mit dem zu tun, was ich in Antwort auf Herm Sachs gesagt habe. Wir haben der Rechtsprechung des Bundesverfassungsgerichts ganz andere Konstitutionalierungstendenzen und -schübe zu verdanken, über die man kritischer nachdenken müsste, als gerade über das, was hier nur schrittweise angedacht worden ist. Wir sehen gerade bei Art. 41 EMRK: Alle möglichen Menschenrechte sind als solche tauglich, Entschädigungsansprüche auszulösen; und was das Beispiel mit der Versammlungsfreiheit angeht: Ich vermag nicht einzusehen, warum jemand, der seine selbstbestimmte Freiheit zur Planung und Organisation einer Versammlung einsetzt, bei deren Verbot nicht wenigstens die für diese Freiheit realisierten Aufwendungen ersetzt bekommt. Das hat nichts mit versammlungsfremden Aspekten zu tun, es ist genau die von ihm selbstbestimmte Freiheit, die er opfert, um die Versammlungsfreiheit zu realisieren; und wenn man ihn daran hindert, dann ist das rechtswidrige Umverteilung von Freiheit, zu dem dieser Staat sanktionslos nicht in der Lage sein sollte.

Ich danke damit allen, danke nicht zuletzt dem Vorstand für die „Zwangsverpflichtung“, mich - für mich jedenfalls gewinnbringend - mit einem Thema zu beschäftigen, mit dem ich mich freiwillig nie und nimmer beschäftigt hätte. Vielen Dank.

E历guth: Danke schön. Sechs Punkte seien in aller Kürze angesprochen. Zunächst scheint es eine Konstante in der Diskussion über das Ver- 
hältnis von europäischem und deutschem Rechtsschutzkonzept zu geben, eine Konstante dahin gehend, das hat auch der heutige Tag gezeigt, dass wir national über eine hohe Kontrolldichte verfügten. Mir ging es darum, dies in meinem Vortrag - das sei noch einmal betont - für wichtige Bereiche des öffentlichen Rechts zu widerlegen. Überall dort, der gestrige Tag, gerade das Referat von Herrn Alexy bildet einen auch verfassungsrechtlichen Beleg, überall dort, wo es Entscheidungsspielräume gibt, erweist sich eine weit zurückgenommene Kontrolldichte. Insoweit gibt es daher nichts zu verlieren oder aufzugeben.

Zweitens: Der Kompensationsgedanke, der gerade im Verhältnis von Primär- und Sekundärrechtsschutz verstärkt eingebracht wird, dürfte in seiner allgemeinen Berechtigung keineswegs so überzeugend sein, wie das vielfache hiervon Gebrauchmachen nahe legt; dies stellt freilich ein Thema für sich dar. Wenn aber mit dem Kompensationsgedanken gearbeitet wird, dann gilt es sehr darauf zu achten, dass die angebotene Kompensation auch wirklich einen Ausgleich darstellt. Hier liegen, wie im Vortrag angedeutet, die Probleme.

Ferner ist die - zentrale - Frage gesetzlicher Strukturentscheidungen aufgeworfen worden. Aus der Sicht meiner Darstellung, die nur exemplarisch und damit weit über das Planungs- oder Umweltrecht hinaus gerichtet war, eben Entscheidungsstrukturen mit Dezisionsfreiräumen betraf, haben wir es mit einer großen Nähe zwischen deutscher und europäischer Rechtsschutzkonzeption zu tun; zumindest ist die Unsrige hinreichend flexibel, um ohne große Strukturentscheidungen auf die Herausforderungen des Europarechts reagieren zu können. Dazu gehört die Stärkung der Kontrolle von Verfahrenspositionen, wobei ich versucht habe zu verdeutlichen, dass sich hinter bestimmten, keineswegs seltenen Verfahrensrechten materielle Rechtspositionen verbergen, genauer gesagt: in ihnen stecken. Das eben sollte in der Wendung von der Janusköpfigkeit des Verfahrensrechts zum Ausdruck kommen. Dann aber bedarf es keiner oder allenfalls sparsamer parlamentarischer Strukturentscheidungen.

Des weiteren möchte ich mich vor dem Anwurf, das Verhältnis Primärrechtsschutz - Sekundärrechtsschutz sei gerade hinsichtlich der Subsidiarität unzulänglich behandelt worden, verwahren. Dies war Ausgangspunkt und systembildendes Element meines gesamten Vortrags.

Nun zur Kritik am Rechtsschutz auf vorgelagerter Ebene. Natürlich gibt es nicht überall derart vorgelagerte Ebenen, aber es gibt sie in weiten - und wichtigen - Bereichen des öffentlichen Rechts; auch war der Blickwinkel dahin gehend zu öffnen, dass es nicht nur um vorgelagerte Entscheidungen mit rechtlicher Bindungskraft gehen darf, sondern auch um programmatische Vorentwürfe, von denen ebenfalls Wirkungen aus- 
gehen. Was die Frage der Abschichtung selbst anbelangt, so sind die Handlungsspielräume, dies im Materiell-Rechtlichen zu belassen und nicht verwaltungsprozessual einzubinden, denkbar gering. Die jüngere nationale Gesetzgebung hat hier prozessuale Angriffsmöglichkeiten auf jeden Fall von Vorhabenträgern geschaffen, das Gemeinschaftsrecht geht jetzt noch weiter, indem nicht nur besagte Umweltprüfung auf vorgelagerter Ebene als solche vorgeschrieben, sondern auch verwaltungsprozessualer Rechtsschutz gefordert wird, der national vorzusehen ist. Daran werden wir nicht herumkommen - und ich meine, dass solches in der Konsequenz der Entwicklung liegt.

Schließlich: Wie integrierter Umweltschutz im Gefolge des Artikelgesetzes zur UVP-Änderungsrichtlinie und anderen Richtlinien umzusetzen sein wird, ist natürlich auch ein Thema für sich. Ich meine allerdings nicht, dass hier der Fehler auf gemeinschaftsrechtlicher Ebene zu suchen ist. Zwar handelt es sich um einen weiten und vielleicht auch vagen Begriff. Nur bilden Erkenntnisse der Naturwissenschaften seinen Hintergrund, die damit Eingang in die Gesetzgebung gefunden haben. Überdies ist der Begriff keineswegs gänzlich konturenlos. Mit dem Kommissionsentwurf zum UGB liegen durchaus vollziehbare Vorschläge vor, u.a. dergestalt, im Wege von Planfeststellungsverfahren, i. ü. mit erweiterter Konzentrationswirkung die Dinge vom Rechtlichen her in den Griff zu bekommen. Danke.

Vorsitzender: Vielen Dank, Herr Erbguth, und nochmals ganz herzlichen Dank an alle vier Referenten, Dank aber auch an Sie alle für den intensiven wissenschaftlichen Gedankenaustausch. Die Aussprache zu unserem zweiten Beratungsgegenstand ist damit abgeschlossen, und ich darf den Vorsitz an Herrn Frowein übergeben.

Vorsitzender (Frowein): Ich möchte den Referenten dieser Tagung und allen denjenigen, die teilgenommen haben und aktiv mitgestaltet haben, sehr herzlich danken. Vor allem aber möchte ich denjenigen danken, von denen wir nur wenige gesehen haben; die uns diese Möglichkeit durch Organisation, Planung und laufende Überwachung ermöglicht haben, als erster steht dafür natürlich Herr Knemeyer, den wir gesehen haben, und dem wir ganz besonders danken. Aber hinter Herrn Knemeyer stehen viele junge Damen und Herren, wir haben sie gelegentlich mal mit einem Augenwinkel gesehen, aber kaum richtig bemerkt; aber ich denke, jeder, der so etwas schon einmal organisiert hat, der weiß, wie wichtig diese Tätigkeit ist und wenn Sie so freundlich sind, Herr Knemeyer, unseren Dank zu übermitteln und damit, das sollte man vielleicht auch ruhig mal laut sagen, die Einladung zu einem von Ihnen gestalteten Abendessen, zu dem 
Sie auf Kosten der Staatsrechtslehrervereinigung alle diejenigen, die daran so intensiv mitgewirkt haben, in unserem Namen einladen. Ich glaube, es steht uns in dieser schönen Stadt und ihrer Umgebung noch sehr Schönes bevor. Ich hoffe, es werden doch noch eine ganz Reihe von uns daran teilnehmen, sowohl heute Abend als morgen auf dem Ausflug. Dann vertagen wir uns auf St. Gallen, aber ich möchte diese letzte Erscheinung vor dem schon etwas gelichteten Kreis der gesamten Vereinigung nicht beenden, ohne auch meinen beiden Vorstandskollegen, Herrn Ipsen und Herrn Bauer, sehr herzlich zu danken für die Zusammenarbeit in diesen letzten zwei Jahren. Sie werden gemerkt haben, dass wir in einer stark traditionsbestimmten Vereinigung ein paar kleine Tüpfelchen verändert haben, und ich hoffe sehr, dass diese Veränderungen Wirkung auch für die Zukunft haben mögen. Ich hoffe, dass der eine oder andere auch den Eindruck hat, dass sie sinnvoll sind. Wir sind davon sehr überzeugt, und ich hoffe, dass wir insofern einen kleinen Beitrag zu der erfolgreichen Geschichte der Vereinigung der Deutschen Staatsrechtslehrer geleistet haben. 